\title{
Current state of pediatric cardiac transplantation
}

\author{
Anne I. Dipchand \\ Labatt Family Heart Centre, Hospital for Sick Children, University of Toronto, Toronto, Canada \\ Correspondence to: Anne I. Dipchand, MD. Hospital for Sick Children, 555 University Avenue, Toronto, Ontario L1V 1L1, Canada. \\ Email: anne.dipchand@sickkids.ca.
}

Pediatric heart transplantation is standard of care for children with end-stage heart failure. The diverse age range, diagnoses, and practice variations continue to challenge the development of evidence-based practices and new technologies. Outcomes in the most recent era are excellent, especially with the more widespread use of ventricular assist devices (VADs). Waitlist mortality remains high and knowledge of risk factors for death while waiting and following transplantation contributes to decision-making around transplant candidacy and timing of listing. The biggest gap impacting both waitlist and overall survival remains mechanical support options for infants and patients with single ventricle physiology. Though acute rejection has decreased progressively, both diagnosis and management of antibody-mediated rejection has become increasingly challenging and complex, as has the ability to understand the implication of anti-HLA antibodies detected both pre- and post-transplantation-including when and how to intervene. Trends in immunosuppression protocols include more use of induction therapy and steroid avoidance or withdrawal protocols. Common long-term morbidities include renal insufficiency, which can be mitigated with surveillance and renal-sparing strategies, and infections. Functional outcomes are excellent, but significant psychosocial challenges exist in relation to neurodevelopment, non-adherence, and transition from childcentered to adult-centered care. Cardiac allograft vasculopathy (CAV) remains a barrier to long-term survival, though it is more apparent that objective evidence of an impact on the allograft is important with regards to impact on outcomes. Retransplantation is rare in pediatric heart transplant recipients. Pediatric heart transplantation continues to evolve in order to address the challenges of the diverse group of patients that reach end-stage heart failure during childhood.

Keywords: Heart transplant; outcomes; sensitization; waitlist; mechanical support

Submitted Oct 04, 2017. Accepted for publication Jan 06, 2018.

doi: $10.21037 /$ acs.2018.01.07

View this article at: http://dx.doi.org/10.21037/acs.2018.01.07

\section{Introduction}

Pediatric heart transplantation continues to evolve in order to address the challenges of the diverse group of patients that reach end-stage heart failure during childhood. This keynote article strives to address some of the contemporary key topics in pediatric heart transplantation. Some important topics are covered elsewhere in this special issue in more detail and the reader is encouraged to refer to these excellent sources for a review of indications for transplantation, mechanical circulatory support/ ventricular assist devices (VADs), donor selection, donation after cardiac death, heart retrieval and preservation, the transplant operation, early graft failure, and featured articles on neonatal transplantation, single ventricle patients, and adult congenital patients.

\section{Data sources/registries and collaborations}

The small numbers of pediatric patients that approach end stage heart failure and follow a trajectory towards heart transplantation continue to challenge us with regards to acquiring the information to develop evidence-based or best practices and/or to study the effect of any new diagnostic or therapeutic strategies on outcomes. Throughout this brief review of the current state of pediatric heart transplantation, 


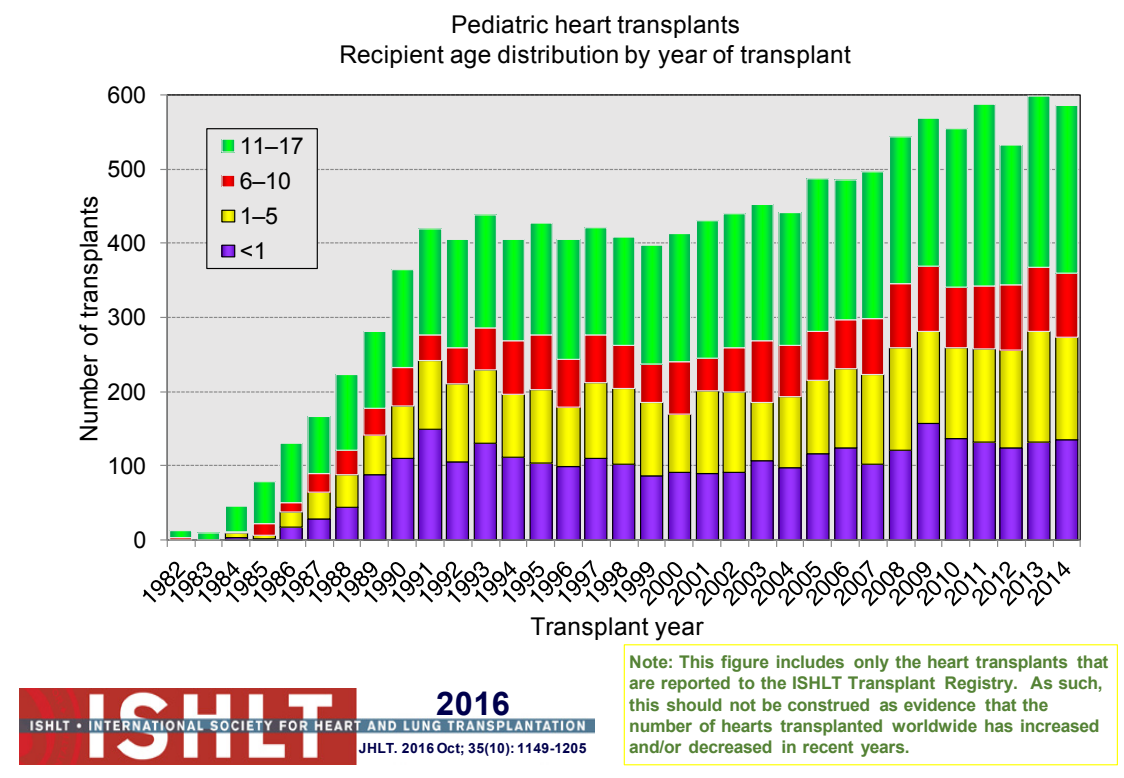

Figure 1 Total number of pediatric heart transplant recipients by age and year of transplant (from the registry of the International Society of Heart and Lung Transplantation. J Heart Lung Transplant 2016;35:1185-95).

factors affecting the ability to carry out clinical and/or observational trials, either prospectively or retrospectively, in this patient population will be highlighted.

There are two main sources of multicenter data for children following listing for transplantation and after transplantation, the registry of the International Society of Heart and Lung Transplantation (ISHLT) $(1,2)$, and the Pediatric Heart Transplant Study (3), in addition to single center reports and smaller collaborations. The ISHLT registry is an international registry where federal mandate in the United States requires all data from the United Network of Organ Sharing (UNOS) to be shared with the database, but is otherwise voluntary. It currently contains data on over 14,000 transplants in children worldwide, which is felt to represent three quarters of the worldwide transplant activity (2). The PHTS is a voluntary, research-based and event-driven multicenter registry that was established in 1993 in order to capture data relative to outcomes, and currently includes 7,716 patients listed for transplantation, of which 5,586 patients underwent transplantation, between January 1, 1993, and December 31, 2016 from 53 centers in North America, the United Kingdom and Brazil.

\section{Recipient demographics}

The number of pediatric heart transplants reported to the
ISHLT registry has increased over time with 442 in 2004, 586 in 2014 (Figure 1) and more recently 684 in 2015 (2). The age distribution of the recipients has remained stable since the mid-1990s (Figure 2). Geographical differences exist worldwide. Most centers in Europe average $<10$ transplants per year while centers averaging $>10$ per year make up $60 \%$ of transplants in North America; this is contrast to an average of 1 to 4 transplants performed by the majority of centers elsewhere in the world. Teenagers account for half of the recipients in Europe and other areas of the world, whereas in North America proportionately more infants undergo transplantation (Figure 3).

Primary diagnoses prior to transplantation have evolved over time. Congenital heart disease has remained the commonest underlying diagnosis in infants younger than 1 year of age though the proportion of infant recipients with cardiomyopathy has doubled to almost $40 \%$ in the most recent era (Figure 4). In older patients, cardiomyopathy remains the predominant diagnosis (Figures 5-7). Primary diagnoses also show geographic variation, with congenital heart disease and retransplantation both more common in North America compared with Europe and the rest of the world (Figure 8) $(1,2)$. Congenital heart disease represents a diverse group of diagnoses with a variety of reasons leading to transplantation not related to the traditional "congestive heart failure" paradigm thus impacting 
Pediatric heart transplants

Recipient age distribution (transplants: January 2004-June 2015)
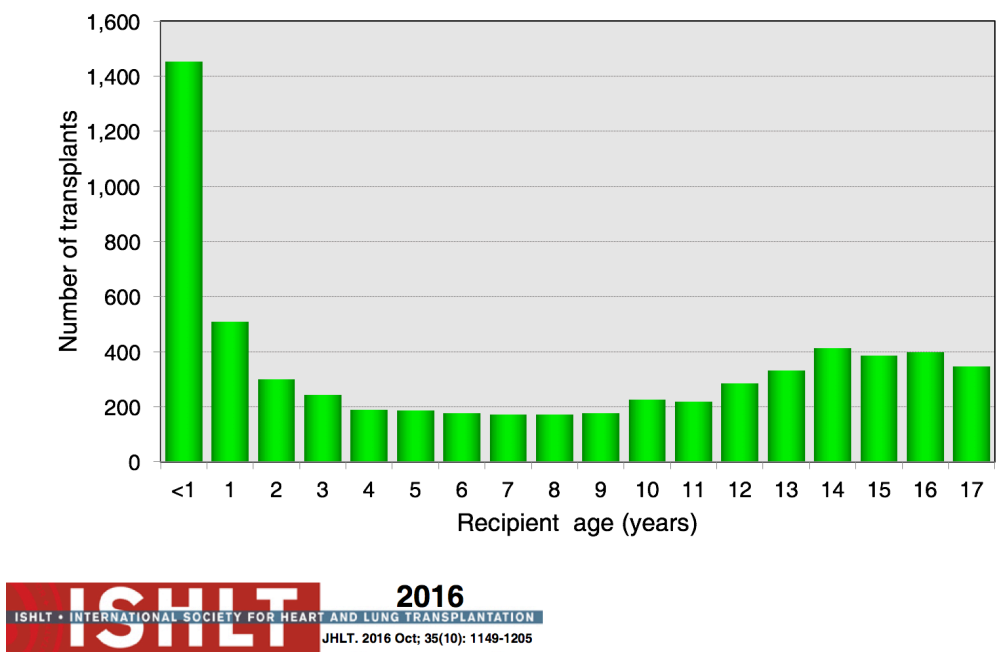

Figure 2 Pediatric heart transplant recipient age distribution January 2004-June 2015 (from the registry of the International Society of Heart and Lung Transplantation. J Heart Lung Transplant 2016;35:1185-95).

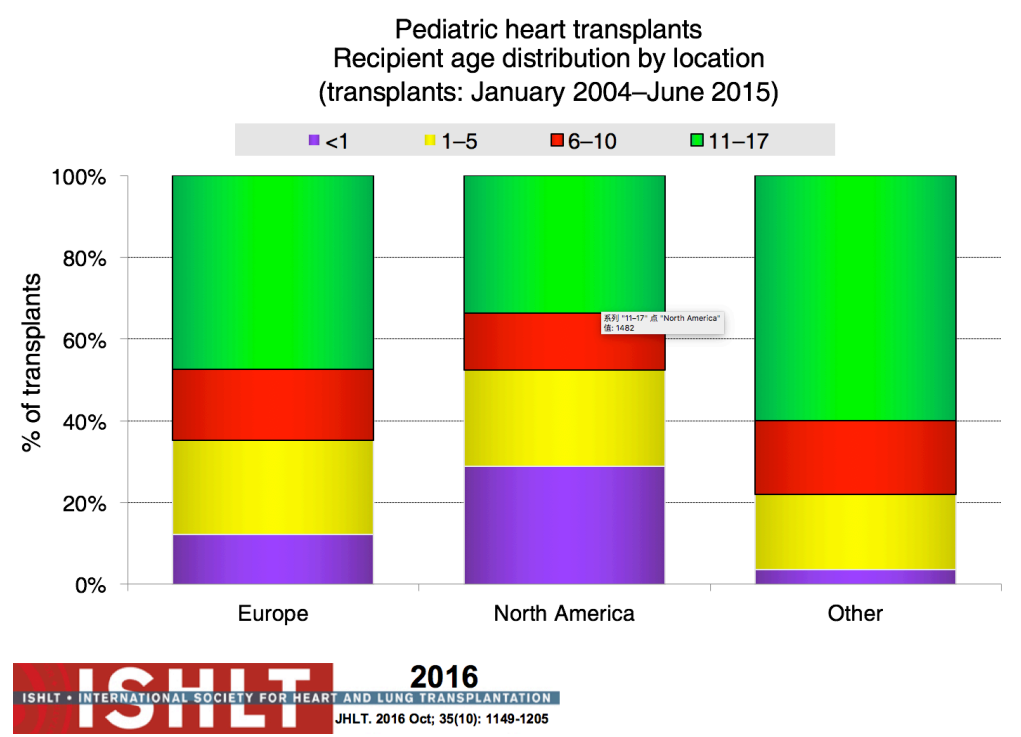

Figure 3 Pediatric heart transplant recipient age distribution by geographic location January 2004-June 2015 (from the registry of the International Society of Heart and Lung Transplantation. J Heart Lung Transplant 2016;35:1185-95).

any registry-based analyses and study trial designs due to the inability to control for the heterogeneity of the patient population. Transplantation in the setting of single ventricles, adult congenital patients, and surgical approaches to unique anatomy are being covered elsewhere in this special edition.

\section{Waitlist mortality}

Mortality for children waiting for heart transplantation continues to be higher than for any other solid organ transplant (4). This is related to a combination of factors including availability of donor organs, the medical state of the recipient, recipient age, weight and diagnosis, and 
Pediatric heart transplants

Recipient diagnosis (age: $<1$ Year)
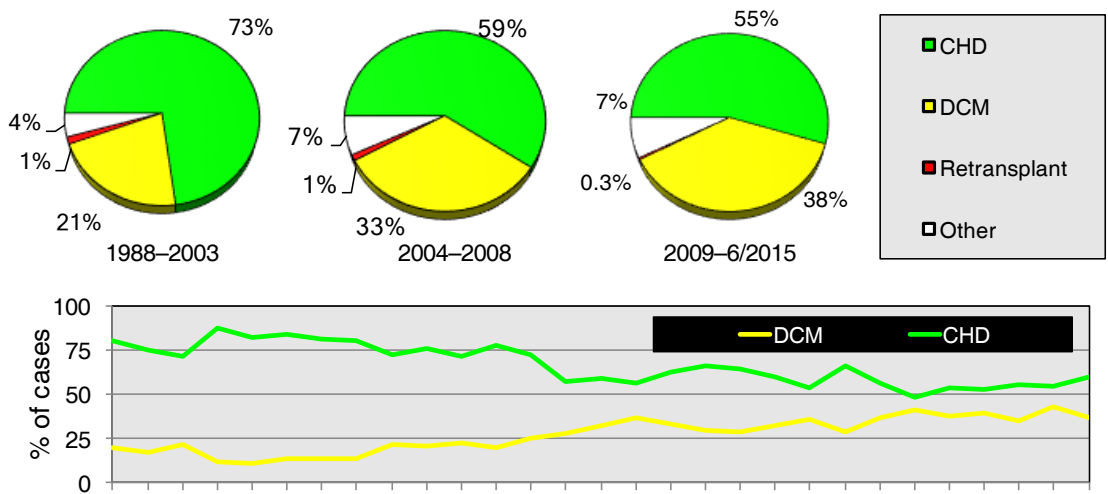

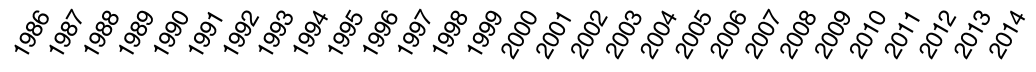

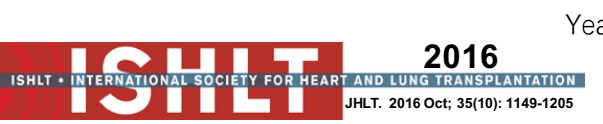

Figure 4 Diagnosis in recipients of pediatric heart transplants aged less than 1 year (from the registry of the International Society of Heart and Lung Transplantation. J Heart Lung Transplant 2016;35:1185-95). CAD, coronary artery disease; CHD, coronary heart disease; DCM, dilated cardiomyopathy.

Pediatric heart transplants

Recipient diagnosis (age: $1-5$ years)

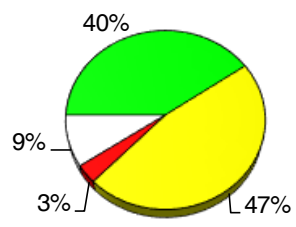

1988-2003
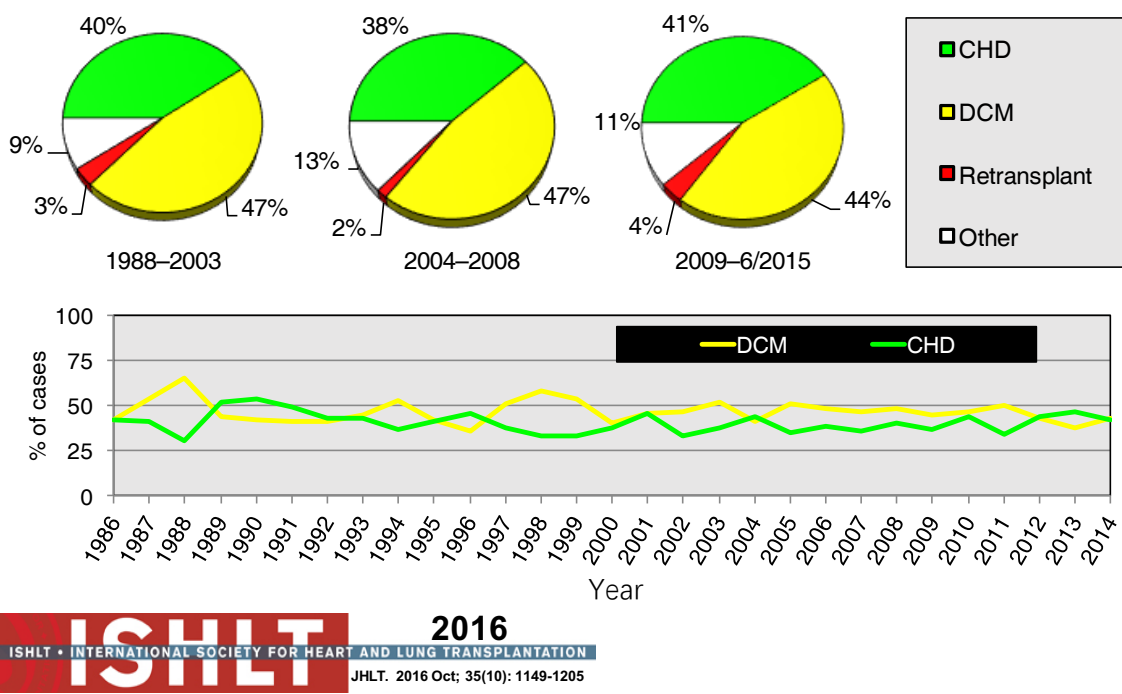

Figure 5 Diagnosis in recipients of pediatric heart transplants aged from 1 to 5 years (from the registry of the International Society of Heart and Lung Transplantation. J Heart Lung Transplant 2016;35:1185-95). CAD, coronary artery disease; CHD, coronary heart disease; DCM, dilated cardiomyopathy. 


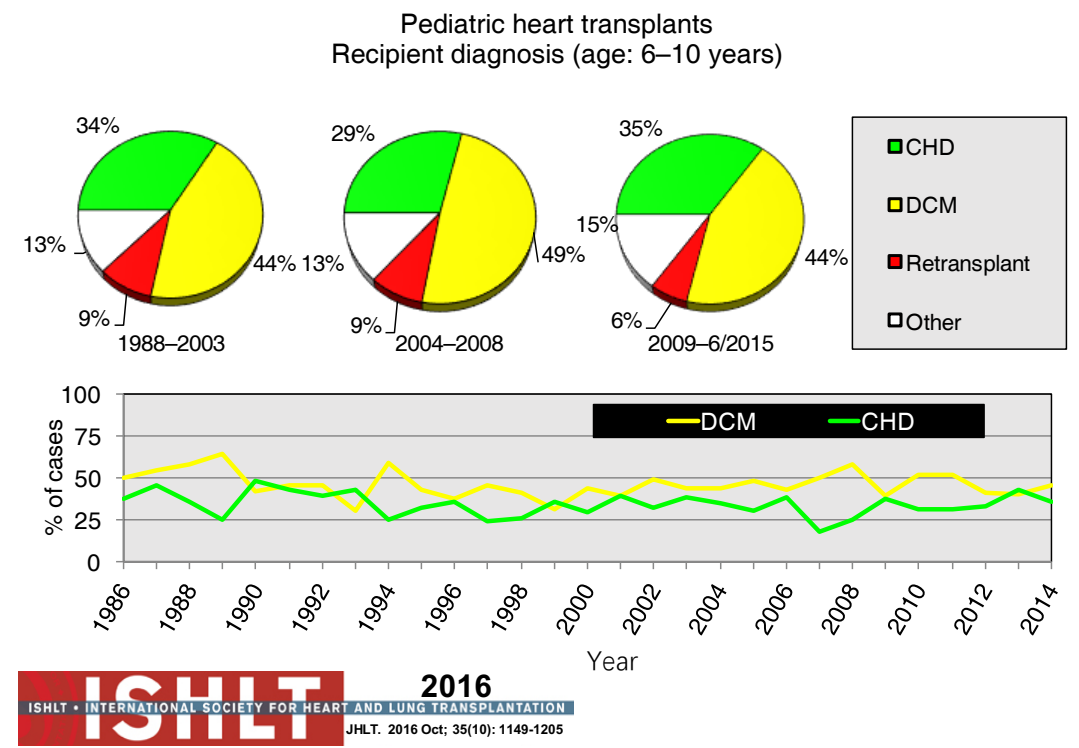

Figure 6 Diagnosis in recipients of pediatric heart transplants aged from 6 to 10 years (from the registry of the International Society of Heart and Lung Transplantation. J Heart Lung Transplant 2016;35:1185-95). CAD, coronary artery disease; CHD, coronary heart disease; DCM, dilated cardiomyopathy.

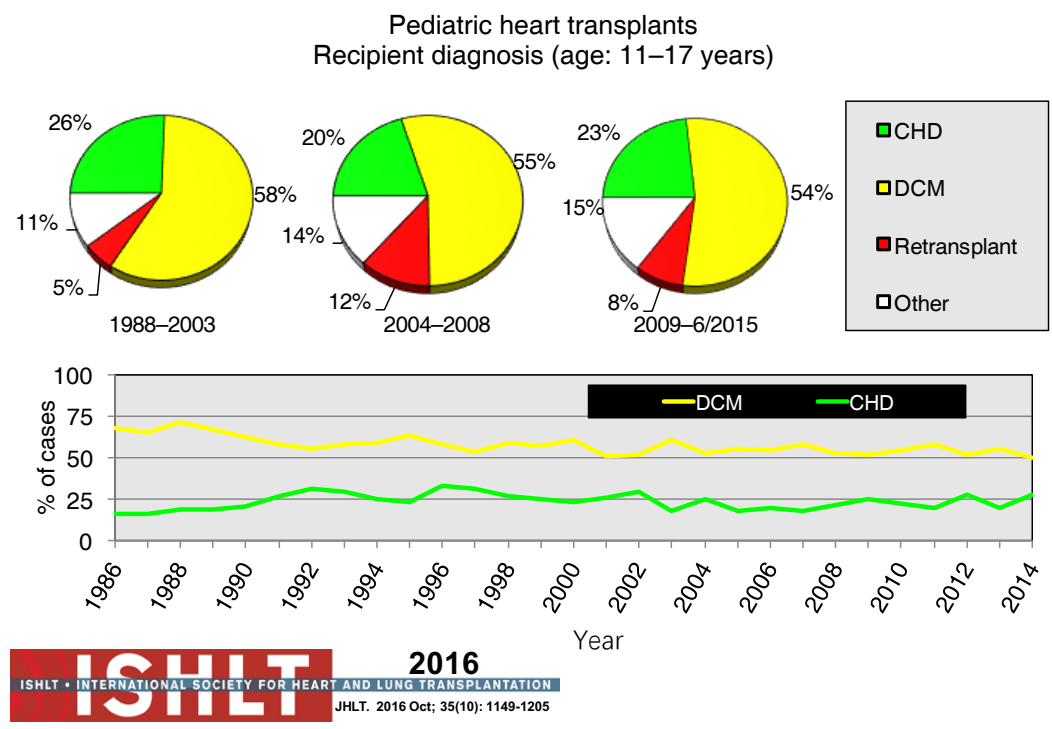

Figure 7 Diagnosis in recipients of pediatric heart transplants aged from 11 to 17 years (from the registry of the International Society of Heart and Lung Transplantation. J Heart Lung Transplant 2016;35:1185-95). CAD, coronary artery disease; CHD, coronary heart disease; DCM, dilated cardiomyopathy.

availability of durable mechanical circulatory support options. Efforts are ongoing to increase donor organ supply including organ donor awareness, countryspecific legislation around organ donation, optimization of donor management and assessment of the heart for transplantation, and novel approaches such as donation after cardiac death which is discussed elsewhere in this special edition. Outside of donor-related approaches, knowledge of factors that influence mortality during the period of waiting plays an important role in decision-making regarding 


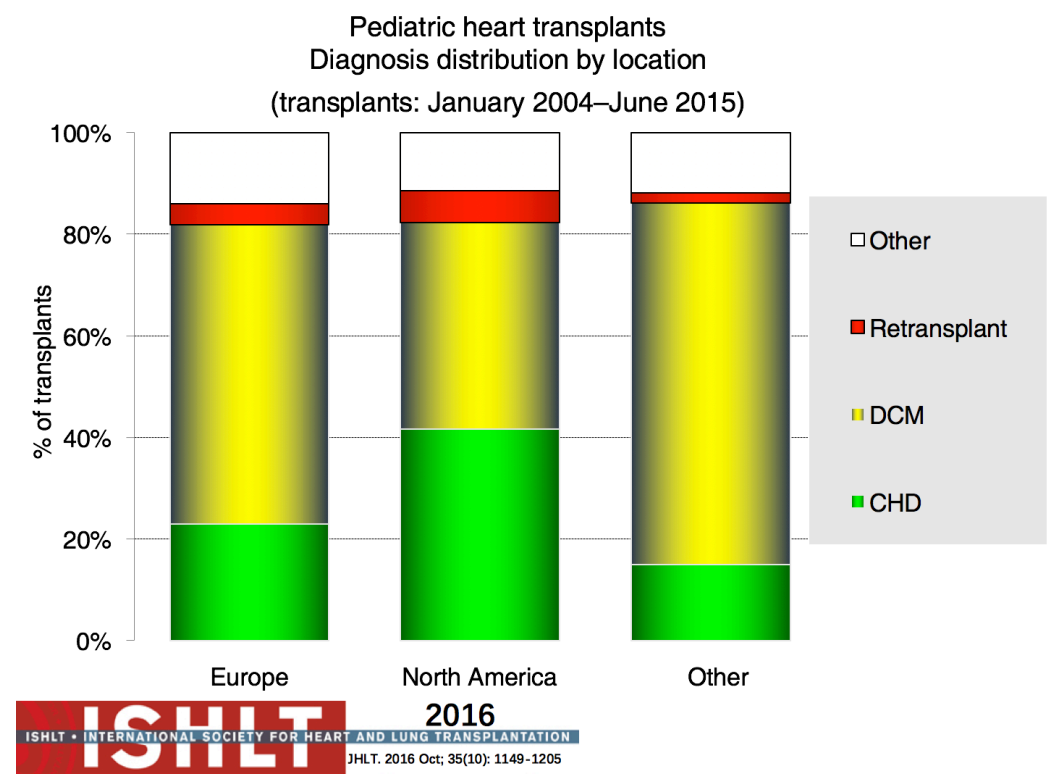

Figure 8 Diagnosis distribution of recipients of pediatric heart transplants by geographic location (from the registry of the International Society of Heart and Lung Transplantation. J Heart Lung Transplant 2016;35:1185-95). CAD, coronary artery disease; CHD, coronary heart disease; DCM, dilated cardiomyopathy.

appropriate timing of listing a patient for transplantation, and stratification of patients listed for heart transplantation.

An analysis of the US Scientific Registry of Transplant Recipients (SRTR) database demonstrated that even though the overall 1-year mortality for all listed patients while waiting was $17 \%$, waitlist mortality varied by as much as 10 -fold based on recipient factors (5-39\%) (4). Recipient characteristics associated with an increased waitlist mortality included extracorporeal membrane oxygenation (ECMO) support, ventilator support, listing status $1 \mathrm{~A}$, congenital heart disease, dialysis support and non-white race. Waitlist mortality for infants was consistently higher than that of older patients, ranging from $25-30 \%$, identifying the group with the most critical donor organ shortage. Patients with cardiomyopathy had better waiting list outcomes than patients with congenital heart disease, a group within which waitlist mortality varied according to underlying diagnosis and previous surgical palliation, especially for those patients with single ventricle physiology $(5-7)$. These observations led to the revision of the UNOS heart waitlist stratification to attempt to better reflect clinical acuity and optimize the allocation of organs to the recipients most in need and less likely to survive waiting (with an acceptable transplant benefit). That being said, in the current era, we are still faced with the smallest patients, who are more likely to have congenital heart disease, having the greatest donor organ shortage and the fewest mechanical support options as a bridge to transplantation. This population is in critical need of targeted efforts to expand the donor organ pool, including donation after cardiac death, and develop miniaturized mechanical support options with variations amenable to use in single ventricle configurations.

Several predictive models have been developed that take into account multiple, coexisting clinical variables/ risk factors to estimate survival; therefore, allowing for risk stratification to assist in decision-making around listing for transplantation and allocation of donor organs to specific recipients to optimize survival both pre- and posttransplant (8-11).

Of note, in this era of more widespread availability and use of VADs, a recent analysis from the UNOS database has shown a $50 \%$ reduction in waitlist mortality with a 4 times higher likelihood of surviving to transplantation (12).

\section{Mechanical support}

The current state of mechanical circulatory support and available devices in pediatrics is covered elsewhere in this special issue. Multiple options exist now due to 


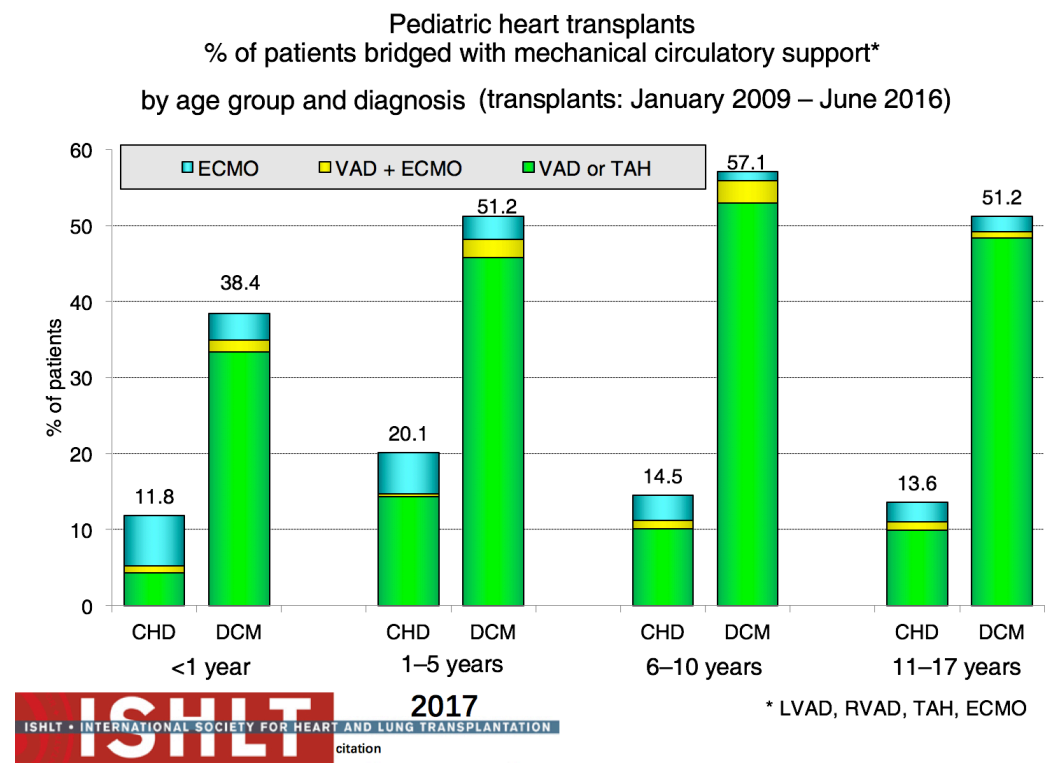

Figure 9 Percent of pediatric heart transplant recipients bridged with mechanical circulatory support by age group and diagnosis January 2009 to June 2016 (from the registry of the International Society of Heart and Lung Transplantation. J Heart Lung Transplant 2017;36:1047-59). CHD, congenital heart disease; DCM, dilated cardiomyopathy; ECMO, extracorporeal membrane oxygenation; TAH, total artificial heart; VAD, ventricular assist device; LVAD, left ventricular assist device; RVAD, right ventricular assist device.

the evolution of mechanical support options including temporary versus durable support and bridge to decision, recovery or transplant, that also allow for crossover between these options. The use of VADs as a bridge to transplant in the pediatric population has continued to increase significantly and currently over $50 \%$ of pediatric patients beyond the infant age group are transplanted from VAD support (2) (Figure 9). These patients can often be mobile and undergo physical and nutritional rehabilitation while awaiting transplantation, which has likely contributed to the reduced waitlist mortality $(12,13)$ without compromising post-transplant survival (Figure 10). However, serious morbidity including stroke, bleeding, infection and device malfunction remains common (14). As depicted in Figure 9, more infants require ECMO support, especially those with congenital heart disease, with the resultant suboptimal outcomes $(13,15)$. Mechanical support options for single ventricle patients and outcomes pre- and post-transplant remain poor (7).

\section{Post-transplant outcomes}

The most recent analysis from the ISHLT registry shows a median survival of 22.3 years for those $<1$ year of age at transplantation, 18.4 years for those 1 to 5 years, 14.4 years for those 6 to 10 years and 13.1 years for those $>11$ yearsnumbers that continue to improve almost annually (Figure 11). Remarkably, survival to 10 years in the most recent era conditional on survival to 1 year post-transplant is now $83 \%$ for all age groups $<10$ years at transplantation $(1,2)$ (Figure 12). The diagnosis before transplantation also affects survival; congenital heart disease continues to be associated with a significantly higher early mortality compared to cardiomyopathy $(2,7)$. Interestingly, the overall survival of cardiomyopathy patients by 3 years posttransplant was $88 \%$ compared to $79 \%$ for patients with congenital heart disease but this difference was negated by 10 years post-transplant with $70 \%$ and $68 \%$ survival respectively (1) (Figure 13). Single ventricle patients with prior staged surgical palliation fare worse with $53 \% 10$-year survival (7). Myocarditis continues to be associated with a worse post-transplant outcome with survival of $83 \%$ at 1 year and $65 \%$ at 3 years post-transplant (16). Some other factors that have been shown to affect 1-year mortality include ECMO, being on a ventilator or dialysis at the time of transplant, other markers of renal insufficiency (creatinine and glomerular filtration rate), recipient body mass index, recipient total bilirubin, ischemic time, and transplant 


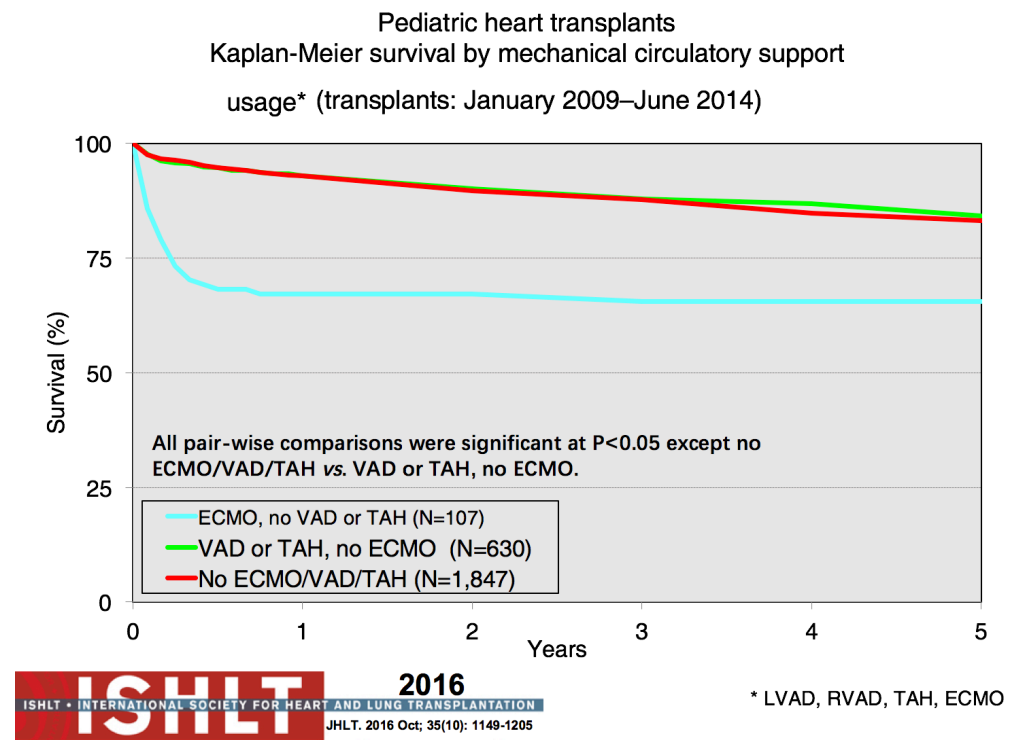

Figure 10 Kaplan-Meier survival in pediatric heart transplant recipients by mechanical circulatory support usage January 2009-June 2014 (from the registry of the International Society of Heart and Lung Transplantation. J Heart Lung Transplant 2016;35:1185-95). CHD, congenital heart disease; DCM, dilated cardiomyopathy; ECMO, extracorporeal membrane oxygenation; TAH, total artificial heart; VAD, ventricular assist device; LVAD, left ventricular assist device; RVAD, right ventricular assist device.

\section{Pediatric heart transplants}

Kaplan-Meier survival (transplants: January 1982-June 2015)
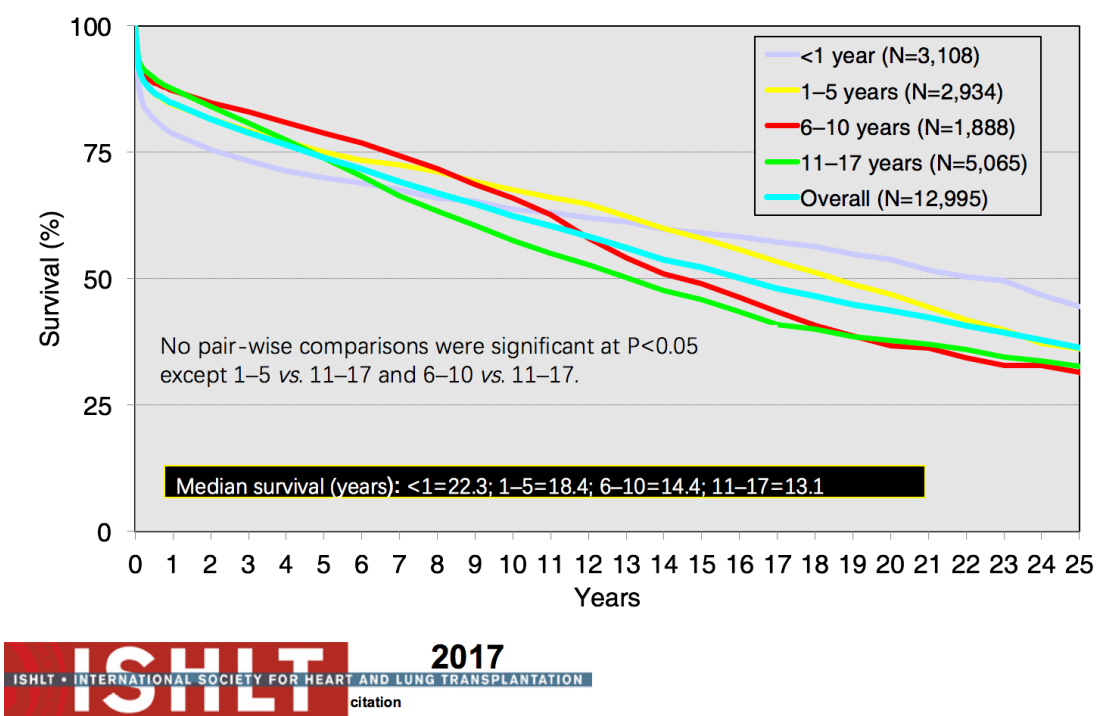

Figure 11 Kaplan-Meier survival curve out to 25 years after pediatric heart transplantation stratified by age at the time of transplantation (from the registry of the International Society of Heart and Lung Transplantation. J Heart Lung Transplant 2017;36:1047-59). 


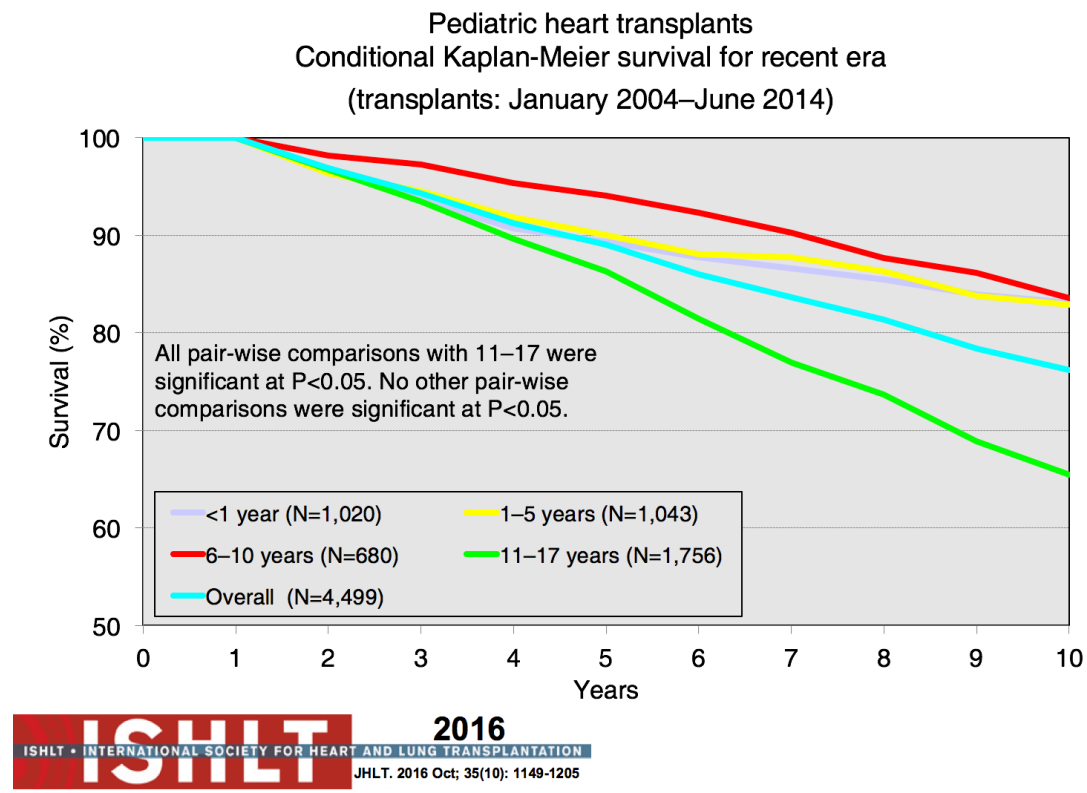

Figure 12 Conditional Kaplan-Meier survival conditional on survival to 1 year after pediatric heart transplant for the most recent era from 2004 through 2014 stratified by age at transplantation (from the registry of the International Society of Heart and Lung Transplantation. J Heart Lung Transplant 2016;35:1185-95).

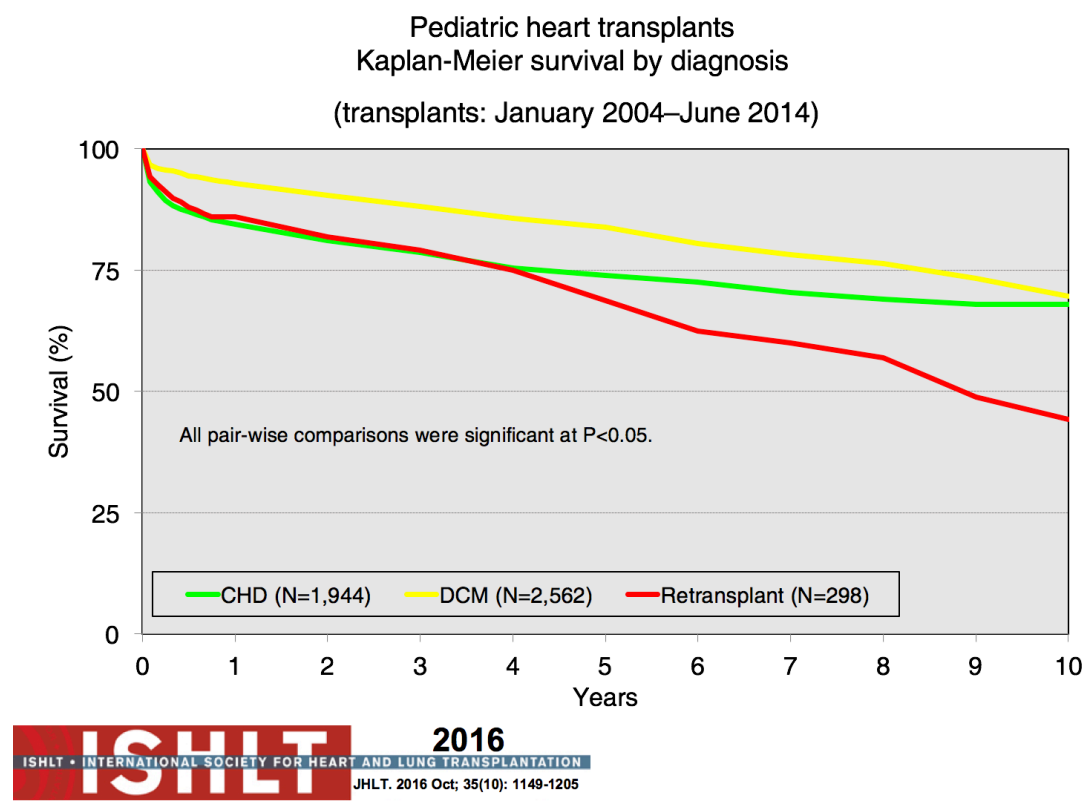

Figure 13 Kaplan-Meier survival for pediatric heart transplant recipients by diagnosis January 2004-June 2014 (from the registry of the International Society of Heart and Lung Transplantation. J Heart Lung Transplant 2016;35:1185-95). CHD, congenital heart disease; DCM, dilated cardiomyopathy. 


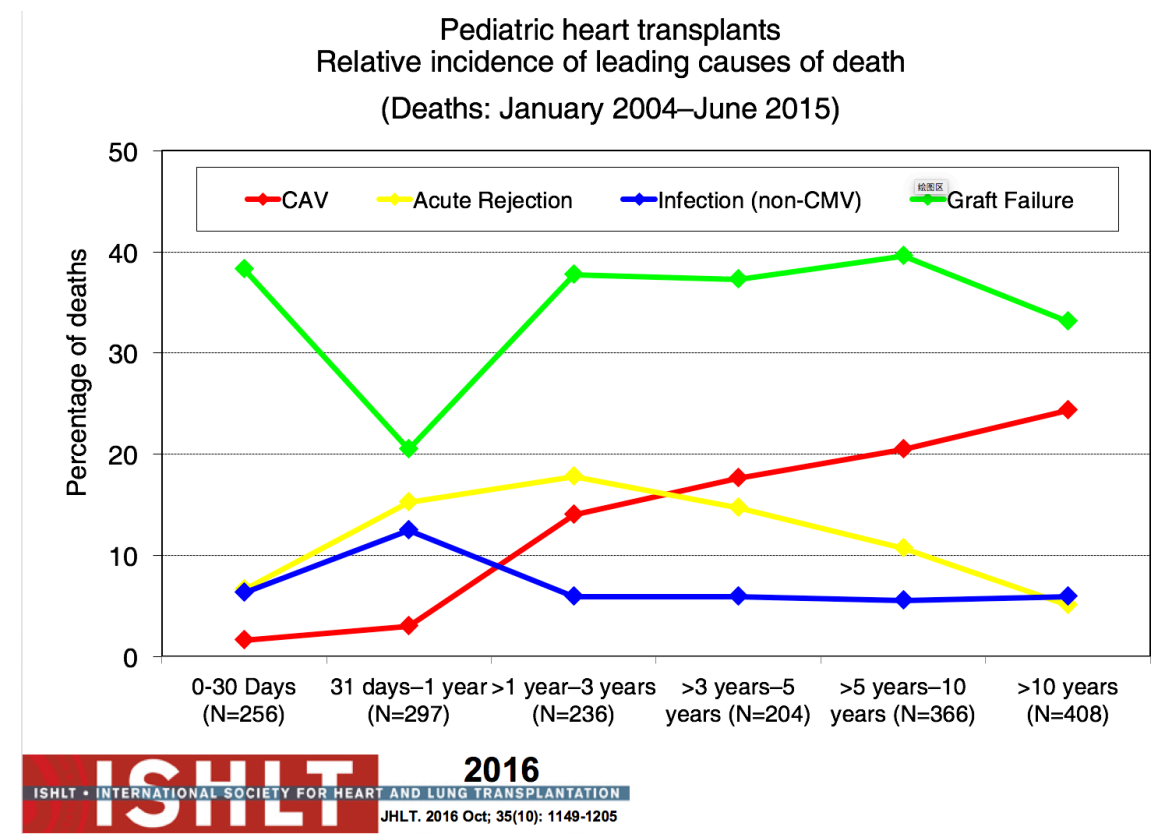

Figure 14 Relative incidence of the leading causes of death for the most recent era from 2004 to June 2015 following pediatric heart transplant (from the registry of the International Society of Heart and Lung Transplantation. J Heart Lung Transplant 2016;35:1185-95). CMV, cytomegalovirus.

center volume (2). Graft failure, rejection, infection, and cardiac allograft vasculopathy (CAV) continue to be the major causes of death within the first 5 years post-transplant (Figure 14).

\section{ABO-incompatible heart transplantation}

Infants continue to experience the best long-term survival following pediatric heart transplantation with a median survival of 20.7 years (Figure 11). There is a belief that this is due in part to the immaturity and malleability of the infant immune system. This has allowed the evolution of ABO-incompatible heart transplantation or transplantation across blood groups (17). In the current era, ABOincompatible heart transplantation is accepted as standard of care-both from a waitlist mortality and a post-transplant survival perspective. Looking at $\mathrm{ABO}$-incompatible listing as a strategy, failure to list for an ABO-incompatible graft and high clinical status emerged as the only factors associated with mortality (18). Multiple publications have now shown equivalent outcomes for $\mathrm{ABO}$-incompatible heart transplantation, including rejection, co-morbidities, CAV, and graft survival (19-21). From an immune perspective, the observations about production of donor- specific isohemagglutinins or lack thereof has provided insight and impetus for further study into mechanisms of B cell tolerance (22). More importantly, experience in both the cardiac and renal literature show that successful ABOincompatible transplants can be undertaken in older patients (and even adults), even when significant isohemagglutinins are present. Risk of significant rejection exists and strategies including plasmapheresis, immunoadsorption, and complement inhibition (eculizumab) may be necessary in order to achieve a reasonable outcome (23).

\section{Sensitization}

Sensitization to human leukocyte antigen (HLA) antibodies depends upon the sensitivity of the testing methodology and is usually related to a medical intervention, especially blood products and homograft tissue used in congenital heart disease repair and VADs. In the past, in the complement dependent cytotoxicity (CDC) test, the serum was tested against a panel of different HLA donors and the likelihood of the serum of the potential recipient having HLA antibodies against a random donor was calculated as a percentage-the panel reactive antibody test (PRA). More recently, solid-phase immunoassay has superseded 
the CDC test for the detection and characterization of HLA antibodies, which is a much more sensitive technique but has resulted in new challenges with respect to the interpretation of donor-specific antibodies (DSA). This test is able to detect HLA antibodies with a high sensitivity but applying this knowledge clinically has proved difficult as the presence of the antibody in and of itself does not mean that it is detrimental to the graft (24).

\section{Donor selection/organ allocation}

Sensitization in the era of solid phase assays now plays a key role in the decision-making around organ allocation and acceptance, predominantly related to the impact on outcomes. The PHTS registry was evaluated for outcomes of sensitized versus non-sensitized recipients and 1 year after listing, of those with a PRA of $<10 \%, 76 \%$ were transplanted and $9 \%$ deceased versus $57 \%$ and $19 \%$ for those with a PRA $\geq 50 \%$. For those that were transplanted, a PRA $<10 \%$ had a $90 \% 1$-year survival versus $73 \%$ with a PRA $\geq 50 \%$ (25). Waiting for a compatible donor increased the risk of dying on the waiting list while accepting a donor to which the recipient was sensitized led to a poorer post-transplant outcome. The requirement for a negative prospective crossmatch increased probability of death waiting but not post-transplant in an analysis of US listings (26). To help decide which is the best approachtake the first organ offered or wait for the best match, a decision model analysis utilizing the OPTN database was undertaken and demonstrated that taking the first available organ offered provided the best outcomes overall (27).

In contrast, the ISHLT registry did not show any impact of an elevated PRA on overall survival (2). In a subset of patients in the PHTS registry with hypoplastic left heart syndrome and staged surgical palliation, post-transplant survival was not affected by sensitization, likely related to current management strategies (7).

Given the importance of sensitization in the current era, the outcomes of transplanting across a positive crossmatch are currently being explored in a prospective, multiinstitutional observational cohort study assessing the impact of pre-transplant sensitization [Clinical Trials in Organ Transplantation in Children (CTOTC), National Institute of Allergy and Infectious Diseases, U01AI077867] $(28,29)$. Sixty percent of patients were sensitized but only $11 \%$ of those had a positive crossmatch. Patients with a positive crossmatch had a higher incidence of antibody mediated rejection (AMR) and acute cellular rejection (ACR) but there was no difference in death, retransplantation or rejection with hemodynamic compromise.

\section{Immunosuppression}

With the ongoing improvement in survival following transplantation, there has been the need to develop protocols and practices to screen for and to minimize morbidities related to chronic immunosuppression in addition to promote graft longevity. There are many centerspecific protocols for maintenance immunosuppression, with collegial debates around the optimal regimen (Figure 15). One interesting observation has been the increase over time in induction therapy, currently used in $71 \%$ of pediatric heart transplant recipients, but without impact on overall survival $(1,2)$ (Figure 16). For the first time, in the most recent era, patients who have received induction therapy have a higher freedom from CAV in the ISHLT registry. A recent PHTS analysis looking at induction demonstrated less rejection in the induction group but no impact on survival on multivariate analysis and an attempt was made to make recommendations based on immunologic risk (30). There was no difference in posttransplant lymphoproliferative disorder or CAV.

Sirolimus or everolimus use in pediatrics remains very low at $<2 \%$ at hospital discharge but increasing to $19 \%$ by year 5 post-transplant (Figure 17). Use is significantly more common in retransplant patients $(28 \%)$ compared to other diagnoses (8-10\%) (1). A recent propensity-matched analysis from the PHTS registry showed sirolimus use in less than $10 \%$ of the patients from 2004 to 2013 with no difference in survival or major adverse events (31). Steroid use has also decreased over time with increasing adoption of steroid withdrawal and steroid avoidance protocols (Figure 18). Reported experience and outcomes remains variable with data from ISHLT showing a worse conditional 1-year survival with maintenance steroid use and data from PHTS showing no difference in 30-day or 1-year rejection or graft survival with a steroid-free regimen (32).

Increasingly being considered is the influence of genetic polymorphisms on the pharmacokinetics of different immunosuppressants and the potential impact on short and long-term outcomes. Identification of potential pharmacogenetic effects on outcomes could influence immunosuppressant choices and has been the focus of a number of multicenter collaborations in pediatric heart transplant recipients (33).

The combination of agents, dosages, and protocols 


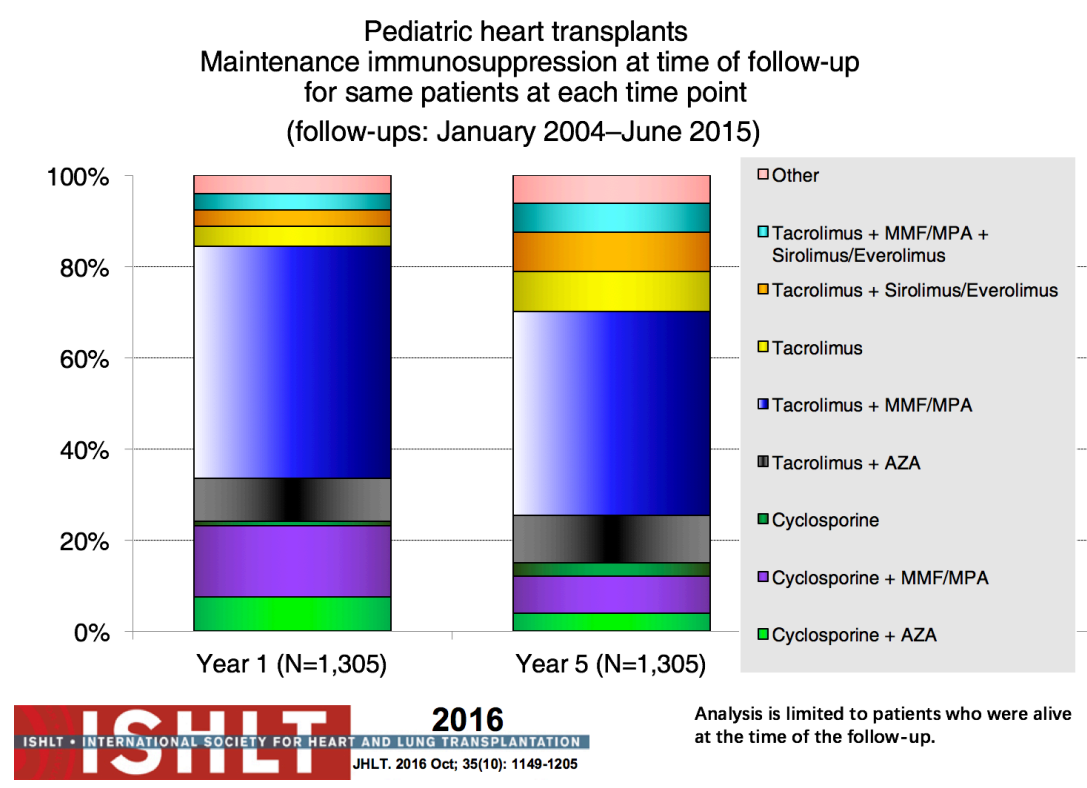

Figure 15 Summary of maintenance immunosuppression in children at 1 and 5 years after heart transplantation (from the registry of the International Society of Heart and Lung Transplantation. J Heart Lung Transplant 2016;35:1185-95). AZA, azathioprine; MMF, mycophenolate mofetil; MPA, mycophenolic acid.

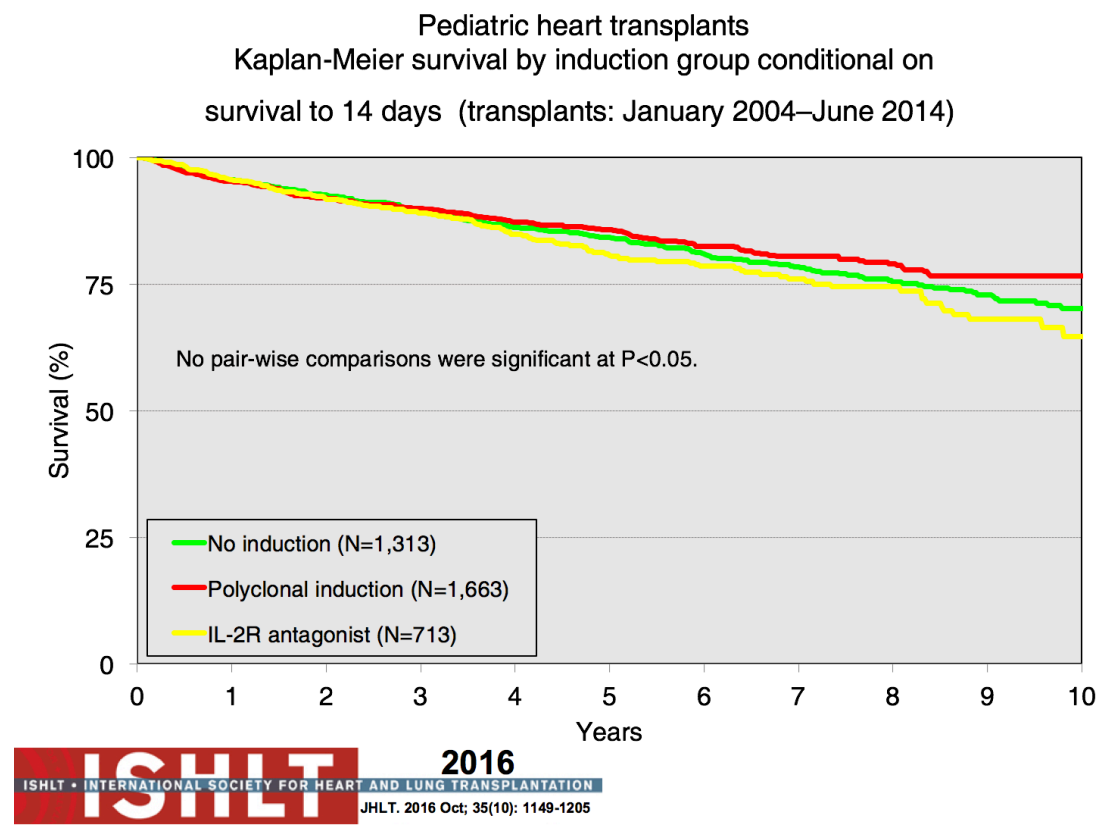

Figure 16 Kaplan-Meier survival by induction group conditional on survival to 14 days in pediatric heart transplant recipients (from the registry of the International Society of Heart and Lung Transplantation. J Heart Lung Transplant 2016;35:1185-95). 
Pediatric heart transplants

Maintenance Immunosuppression at time of transplant

discharge by era (transplants: January 2004-June 2015)

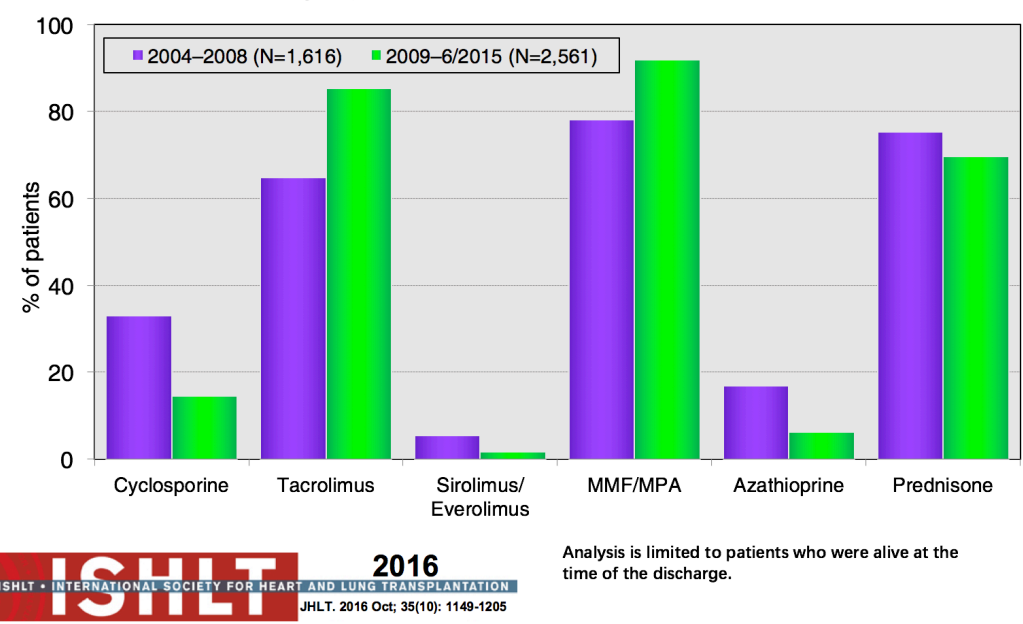

Figure 17 Maintenance immunosuppression at time of transplant hospitalization discharge by era in pediatric heart transplant recipients (from the registry of the International Society of Heart and Lung Transplantation. J Heart Lung Transplant 2016;35:1185-95). MMF, mycophenolate mofetil; MPA, mycophenolic acid.

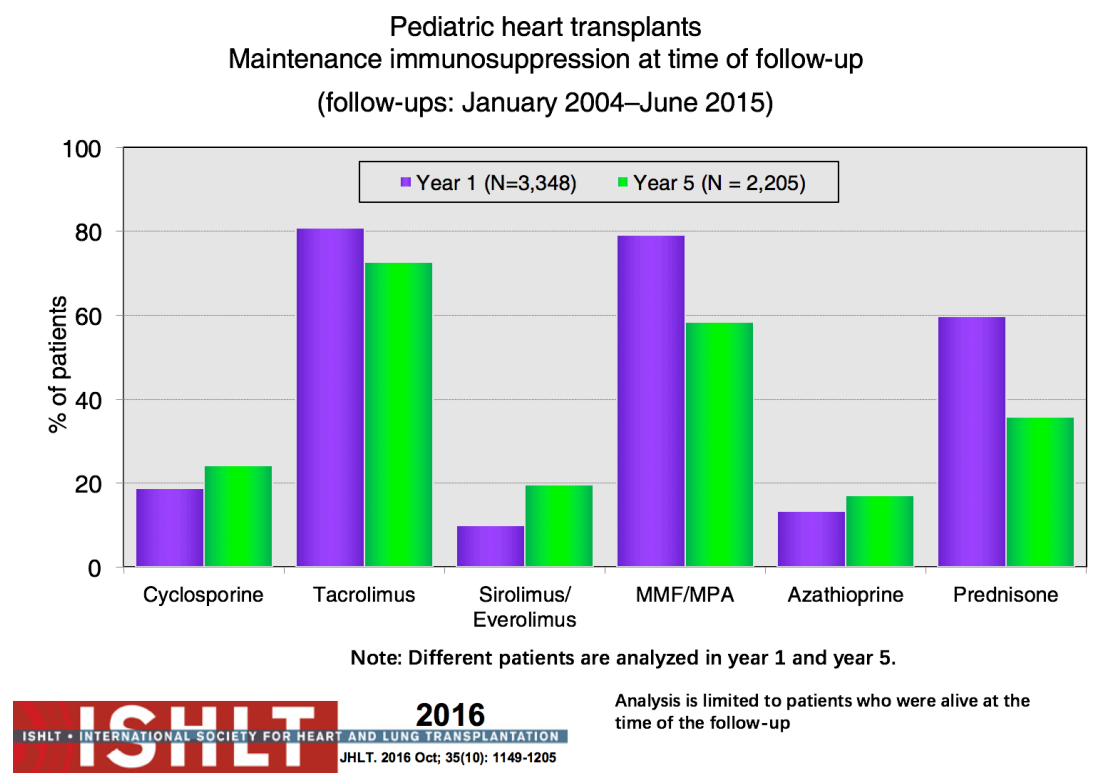

Figure 18 Maintenance immunosuppression at time of 1- and 5-year follow-up in pediatric heart transplant recipients (from the registry of the International Society of Heart and Lung Transplantation. J Heart Lung Transplant 2016;35:1185-95). MMF, mycophenolate mofetil; MPA, mycophenolic acid. 


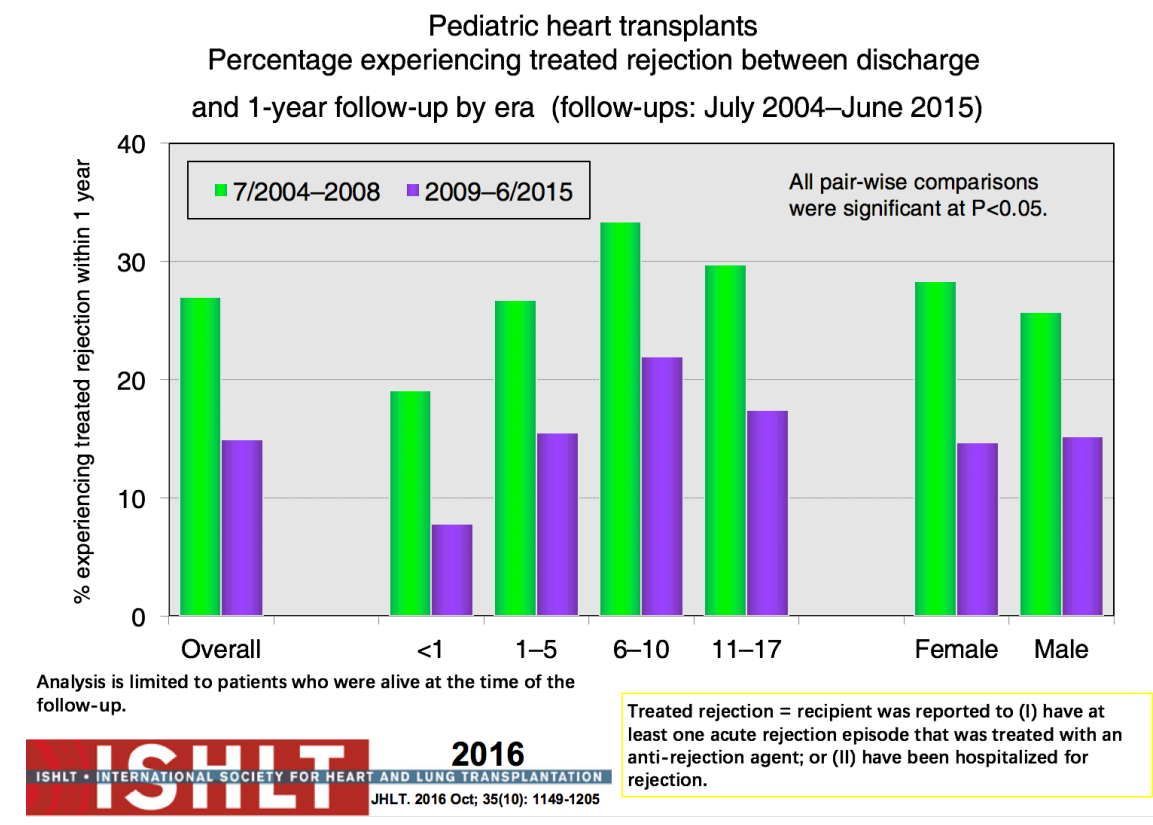

Figure 19 Percentage of pediatric heart transplant recipients experiencing treated rejection between discharge and 1-year follow-up by era (from the registry of the International Society of Heart and Lung Transplantation. J Heart Lung Transplant 2016;35:1185-95).

that are used for both induction and maintenance of immunosuppression make definitive comparisons and recommendations difficult, and add to the challenges with designing clinical trials in this patient population.

\section{DSA post-transplant}

Much still needs to be learned about the significance of either pre-existing or newly detected DSA (ndDSA) posttransplant $(34,35)$. These may be transient in which case, especially if early in the post-transplant course, may be of little significance. However, if they are persistent they are associated with worse graft survival (36). In the CTOTC trial noted above, one third of patients developed ndDSA in the first post-transplant year, mostly within the first 6 weeks, implying that memory responses may predominate over true de novo DSA production. In the absence of pre-transplant DSA, patients with ndDSA had significantly more ACR but not AMR, and there was no impact on graft and patient survival in the first-year post-transplant (37). The more long-term impact of preexisting and ndDSA are being followed in this cohort of patient. Strategies to reduce post-transplant DSA are similar to those pre-transplant, however the indications, necessity and efficacy are as yet unknown.

\section{Rejection}

Acute rejection remains an important cause of mortality and morbidity after transplantation (Figure 14). The risk of rejection is highest in the first-year post-transplant, but data from both the PHTS and ISHLT have shown an overall decline in rejection even in the first-year post-transplant $(1,38,39)$ (Figure 19). This decrease significantly impacts the ability to use rejection as an endpoint in any study design or analysis. The definition of rejection varies and it is often difficult to differentiate ACR and AMR both clinically and in the published literature, further challenging risk factor and outcome analyses and interpretation of therapeutic interventions. There are few identifiable risk factors for rejection prior to transplantation other than sensitization. Both registries show that treated rejection in the firstyear post-transplant decreases long-term survival $(1,40)$ (Figure 20). Myocarditis continues to be associated with death from acute rejection leading to the hypothesis that either immune or infectious mechanisms play a role in the suboptimal outcomes (16).

\section{Surveillance}

Endomyocardial biopsy remains the mainstay for the 


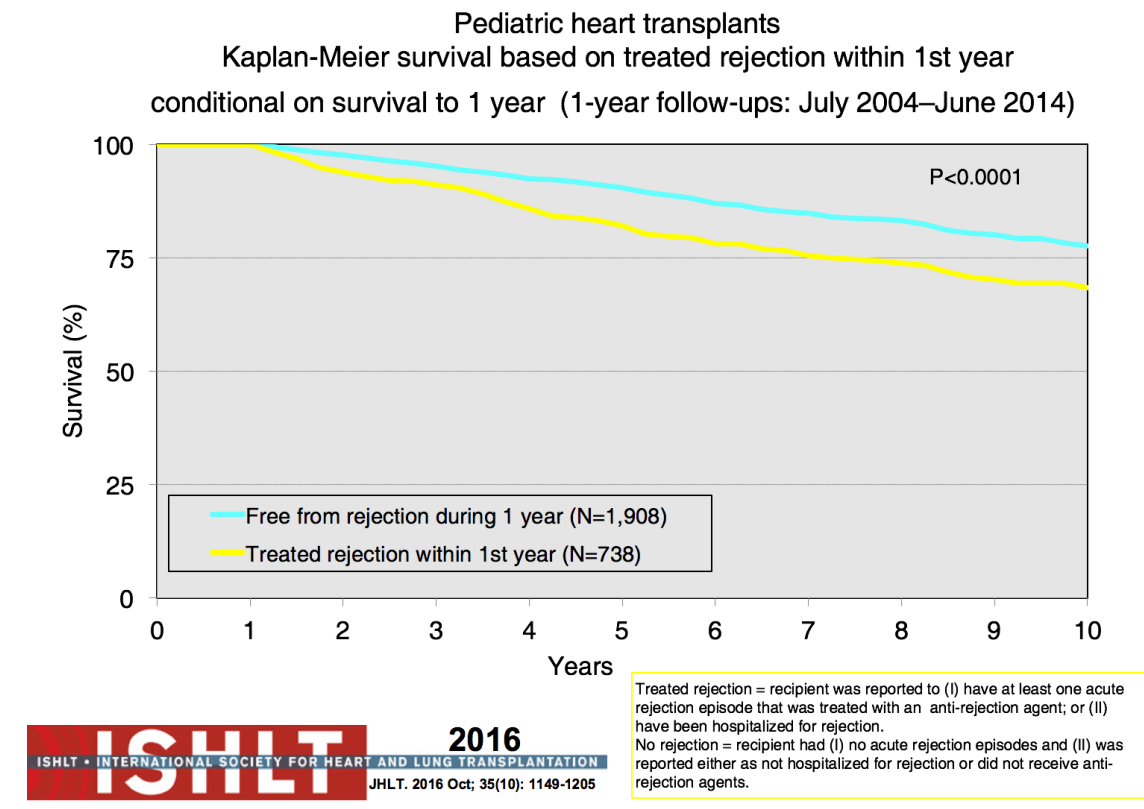

Figure 20 Kaplan-Meier survival in pediatric heart transplant recipients based on the presence of rejection within the first year after transplantation (conditional on survival to 1 year) (from the registry of the International Society of Heart and Lung Transplantation. J Heart Lung Transplant 2016;35:1185-95).

diagnosis of rejection. There are significant differences in the use of routine surveillance biopsies with varying frequencies, centers continuing them indefinitely, others discontinuing them after a period of a few years, and even geographic variation (41). Standardized grading scales from ISHLT exist for biopsy reporting $(42,43)$. For ACR, Grade $0 \mathrm{R}$ indicates no rejection, Grade $1 \mathrm{R}$ mild rejection, Grade $2 \mathrm{R}$ moderate rejection, and Grade $3 \mathrm{R}$ severe rejection. More recently, to aid in the diagnosis and treatment of AMR, ISHLT also developed a pathology-based AMR grading system (43). There have been many efforts to identify and validate non-invasive tests for diagnosing rejection, including echocardiography, intramyocardial electrography, and profiling of gene expression that are beyond the scope of this chapter (44). As yet, no non-invasive test has been developed to consistently and accurately diagnose and/or predict rejection.

\section{Treatment}

Treatment for acute rejection depends on multiple factors including the type, grade, time post-transplant, clinical and hemodynamic effect, maintenance immunosuppression and co-morbidities, and potential co-factors (e.g., infection, non-adherence, etc.). There is general agreement that mild rejection does not require specific intervention. Moderate rejection usually requires some degree of increased immunosuppression, which generally includes an oral or intravenous bolus of corticosteroid, and an increase in maintenance immunosuppression. There remains the question as to whether asymptomatic rejection found on routine surveillance biopsy needs to be treated at all given the lack of evidence for an impact on overall outcomes (45).

\section{Antibody mediated rejection}

AMR in adult recipients has been reported to be associated with CAV and graft failure, but there is a paucity of data in pediatrics. As noted above, the diagnosis of AMR is fraught with challenges, both clinically and from a pathologic perspective. This is further confounded by the evolution of HLA antibody detection technology and the increasing surveillance and detection of DSA as discussed above. Retrospectively assigning the ISHLT pathologic AMR (pAMR) grades in a single center review showed pAMR2 or higher in $18 \%$ of biopsies and $59 \%$ of patients; pAMR3 was associated with worse cardiovascular outcomes (46). Using a cohort of patients from 2010-2014 to look at AMR 


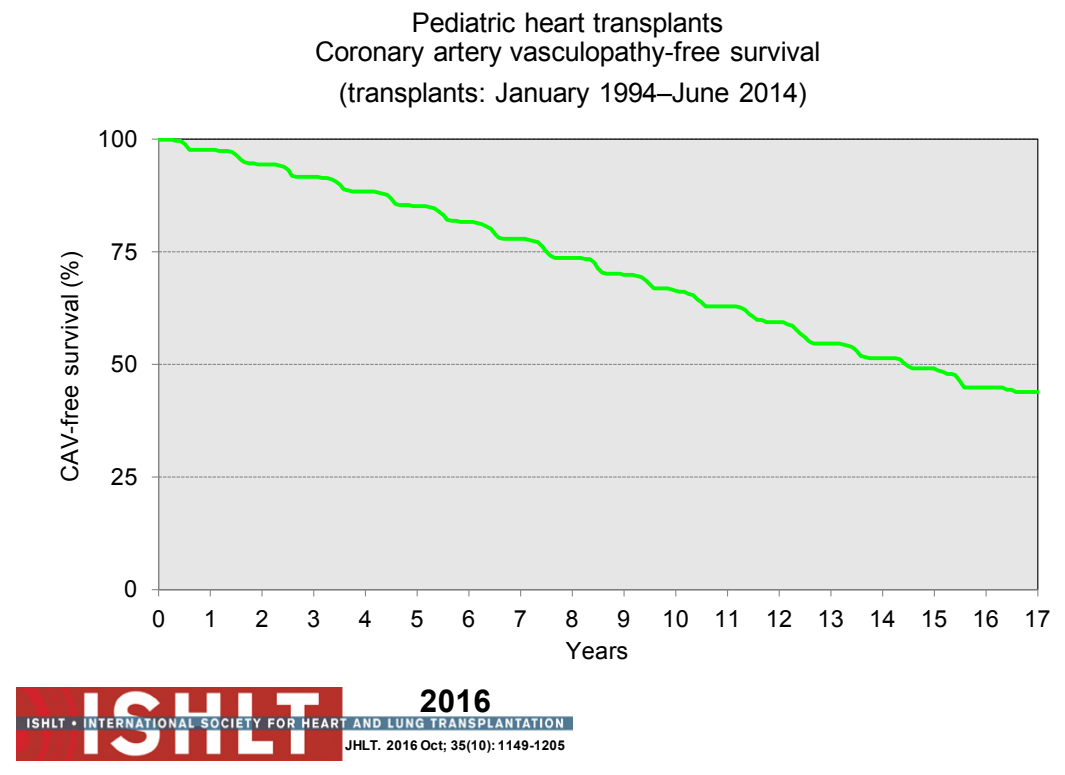

Figure 21 Freedom from cardiac allograft vasculopathy $(\mathrm{CAV})$ in pediatric heart transplant recipients (from the registry of the International Society of Heart and Lung Transplantation. J Heart Lung Transplant 2016;35:1185-95).

in a more recent era, an analysis from PHTS showed an incidence of treated AMR of $12 \%$ in the first 3 years postheart transplant with risk factors including a diagnosis of coronary heart disease (CHD), sensitization, a positive crossmatch, severe ACR, and the use of maintenance steroids (40). Mixed rejection was seen in a total of $30 \%$ of patients with moderate to severe ACR by biopsy grading. Follow up was too short to ascertain an impact on more long-term outcomes. There is general agreement that AMR with graft dysfunction should be treated and treated AMR is associated with worse outcomes. Controversy exists around the optimal approach to asymptomatic biopsy-detected AMR, including whether to treat, frequency of follow up, and surveillance.

\section{Late rejection and rejection with hemodynamic compromise}

Rejection beyond 1 year post-transplant, or late rejection, has also decreased over time, though with a persistent impact on CAV and mortality (47). Risk factors include early rejection, anti-HLA antibodies, older age, AfricanAmerican race, and non-adherence (39). Observations regarding rejection with hemodynamic compromise requiring inotropic support have not revealed the same era effect as with the other types of rejection, and worse survival of $49 \%$ at 5 years persists (39). Hemodynamic supports appropriate for the clinical presentation and aggressive intensification of immunosuppression is required to manage any rejection with hemodynamic compromise.

\section{CAV}

$\mathrm{CAV}$ is a leading cause of death beyond 3 years after transplantation (Figure 14). Risk factors for CAV in children include older donor age, older recipient age, donor cigarette use, recipient black race, transplant era, no induction therapy, re-transplantation, rejection in the first year posttransplant and repeated episodes of cellular rejection $(2,48)$. Freedom from CAV is $40 \%$ by 17 years post-transplant (Figure 21) but does vary by age (Figure 22), and is one of the few things that is impacted upon by induction therapy (Figure 23).

Diagnosis is highly dependent on the type and frequency of surveillance post-transplant which is highly variable. Coronary angiography remains the gold standard but has limitations and provides minimal information on the impact of CAV on allograft function. The consensus ISHLT grading system for CAV was developed both for purposes of standardization and to take into account functional parameters from invasive measurements or surrogate markers of hemodynamics 


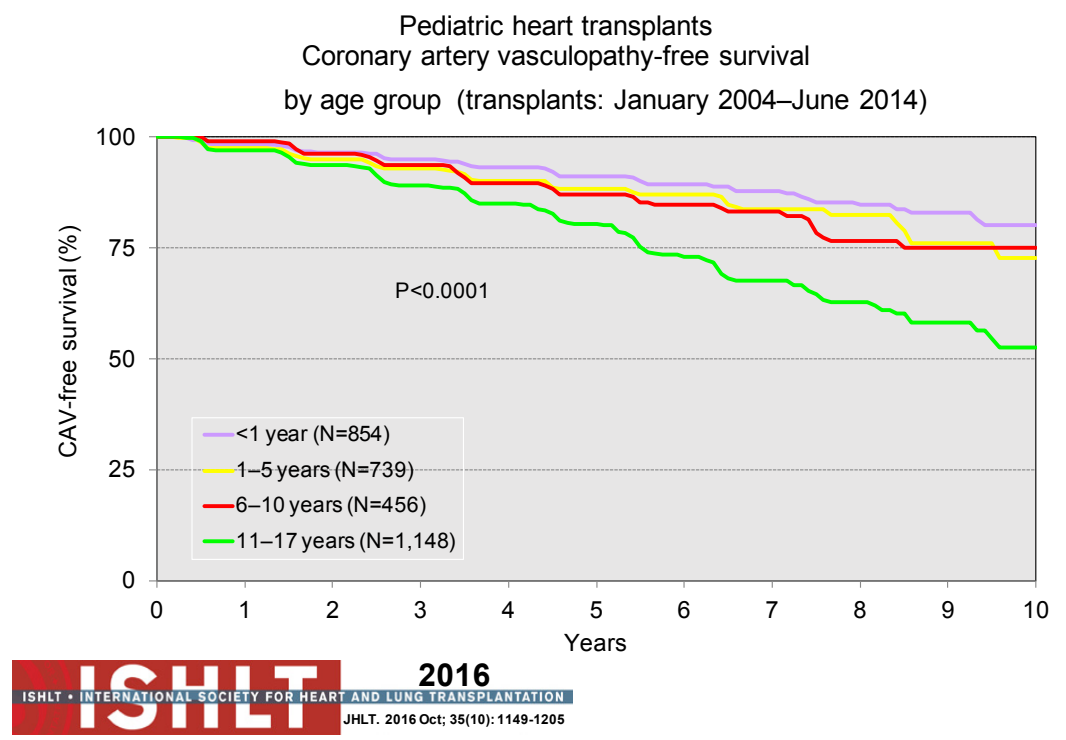

Figure 22 Freedom from cardiac allograft vasculopathy (CAV) in pediatric heart transplant recipients stratified by age group (from the registry of the International Society of Heart and Lung Transplantation. J Heart Lung Transplant 2016;35:1185-95).

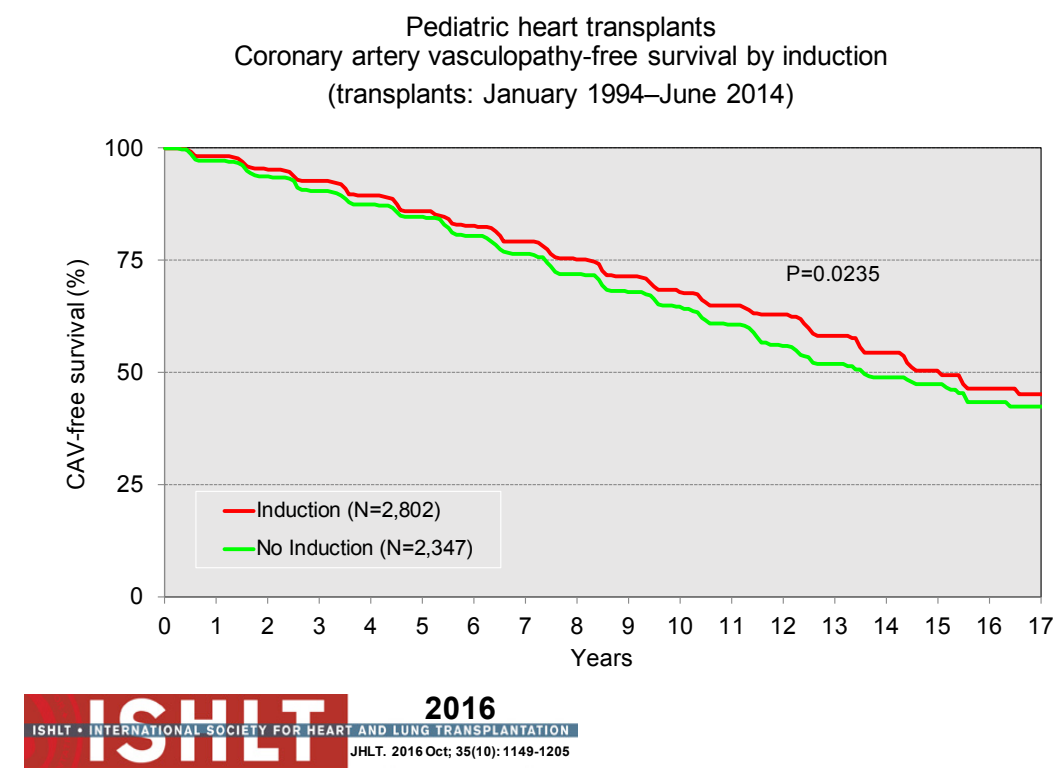

Figure 23 Freedom from cardiac allograft vasculopathy (CAV) in pediatric heart transplant recipients stratified by induction (from the registry of the International Society of Heart and Lung Transplantation. J Heart Lung Transplant 2016;35:1185-95).

from echocardiography (49). Once CAV is evident angiographically, short-term mortality is high (Figure 24); most notably in the infant age group. In a PHTS analysis aimed at validating the ISHLT grading system, an increased risk of graft loss in the setting of CAV was verified with left ventricular ejection fraction $<45 \%$, right atrial pressure $>12 \mathrm{mmHg}$ and/or pulmonary capillary wedge pressure $>15 \mathrm{mmHg}(50)$. 


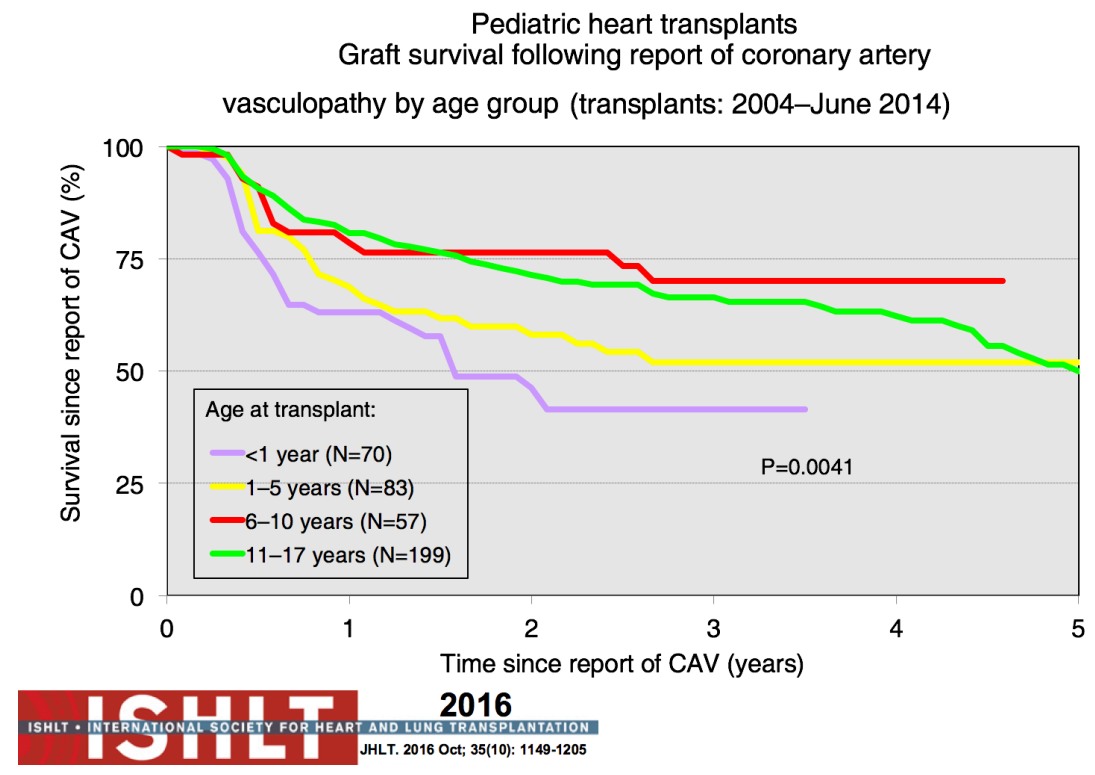

Figure 24 Kaplan-Meier survival following diagnosis of cardiac allograft vasculopathy (CAV) in pediatric heart transplant recipients stratified by age group (from the registry of the International Society of Heart and Lung Transplantation. J Heart Lung Transplant 2016;35:1185-95).

Intravascular ultrasound has been proposed as a better or complementary diagnostic tool for the detection and grading of CAV. Initial optimism has been tempered by technical issues, cost, and lack of meaningful end-points for management and/or prognosis. It is presently being used in a handful of centers on older children due to the size of the available catheters, and most often plays a secondary role (51). MRI is being trialed in a few centers but has yet to reach the level of replacing angiography, either from an imaging perspective or, more recently, microcirculation assessment (52). Optical coherence tomography has been tried in a few centers as a supplementary tool, but has not achieved widespread application in pediatrics (53).

Statins have been shown to play a beneficial role in slowing the development of CAV. Adult studies have clearly shown a benefit of statins on the incidence of acute rejection with hemodynamic compromise, improved 1-year survival, and reduced development of CAV. Two studies in children have shown a lower incidence of CAV during treatment with simvastatin or atorvastatin $(54,55)$, though the PHTS analysis showed no impact on outcomes (56). Despite the paucity of pediatric data, statin use has been incorporated in the majority of maintenance protocols for pediatric heart transplant recipients.

Intervention for established $\mathrm{CAV}$ is challenging, especially in children. The limited experience and reports in the literature make definitive recommendations difficult (57-59). Due to the diffuse nature of the disease, the majority of patients are not amenable to interventional techniques. Ultimately, patients with moderate-to-severe CAV and evidence of graft dysfunction may require consideration for retransplantation.

\section{Retransplantation}

Retransplantation makes up a very small proportion of heart transplants in children annually (albeit with age and geography-related differences) (Figures 5-9); these numbers have decreased over time with less than 20 retransplants reported to the ISHLT Registry in 2014 (1). Given organ donor shortages and ongoing waitlist mortality, controversy remains regarding the role of retransplantation in light of the decreased long-term survival (Figure 25) and increased transplant-related morbidities $(60,61)$. Retransplantation is a risk factor for death at $1,5,10$ and 15 years posttransplant (60). Survival following retransplantation nears that of primary transplantation only when retransplantation occurs at 5 years or more after primary transplantation, compared to shorter time intervals, and this difference remains significant on multivariable analysis (60) 


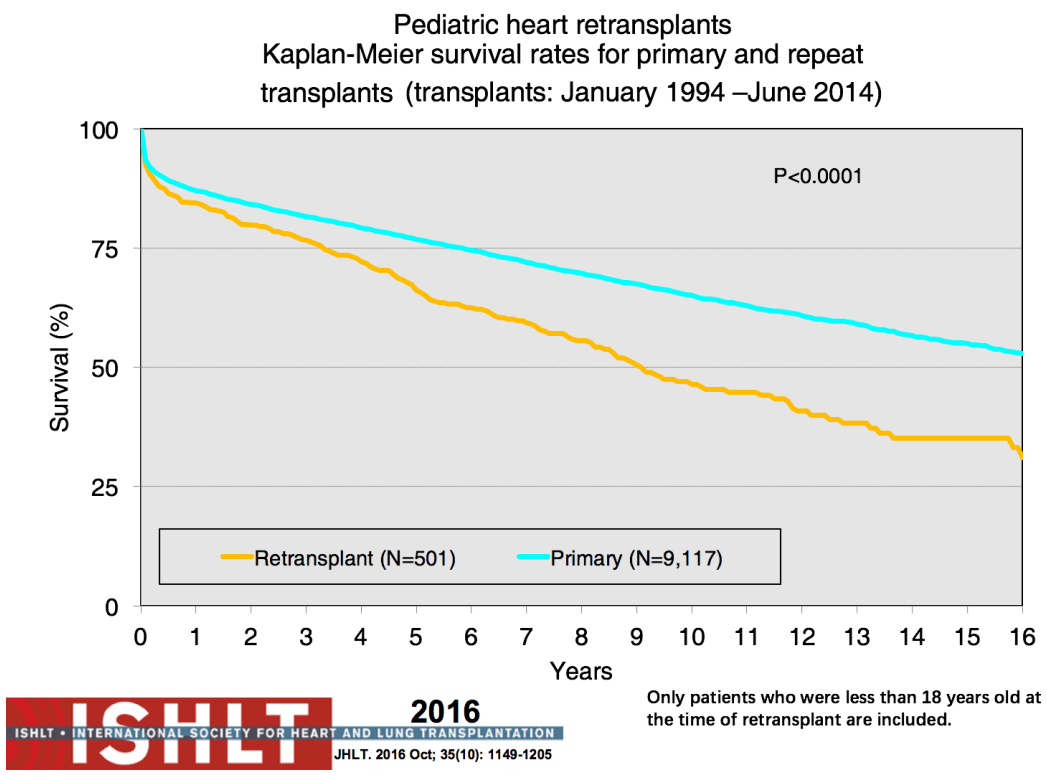

Figure 25 Kaplan-Meier survival curves for primary and repeat transplants in pediatric heart transplant recipients (from the registry of the International Society of Heart and Lung Transplantation. J Heart Lung Transplant 2016;35:1185-95).

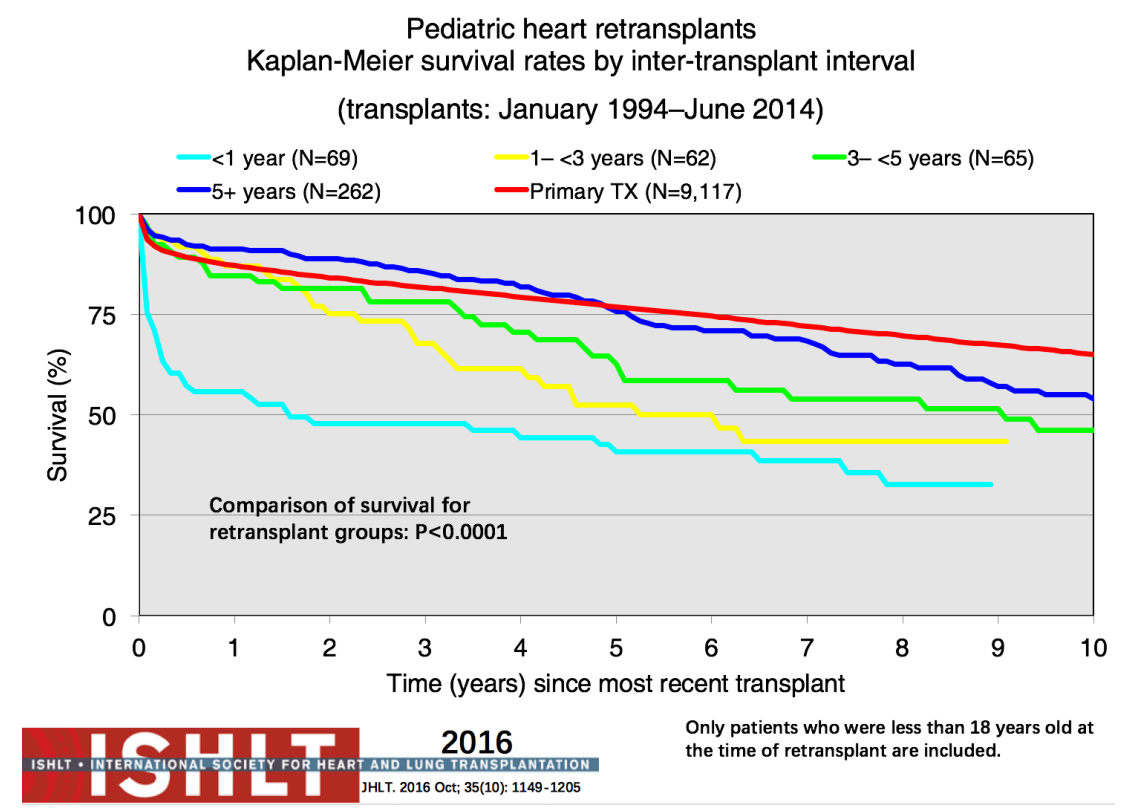

Figure 26 Kaplan-Meier survival for retransplantation in children by intertransplant interval (from the registry of the International Society of Heart and Lung Transplantation. J Heart Lung Transplant 2016;35:1185-95). Tx, transplantation.

(Figure 26). Patients retransplanted for rejection or graft failure, the most common reason within the first year postprimary transplant, have a much lower survival in the first year following retransplantation and serious consideration needs to be given to the candidacy of these patients for retransplantation. Retransplantation for CAV has the highest survival but a lower freedom from CAV in the retransplanted heart and transplant-related morbidities 


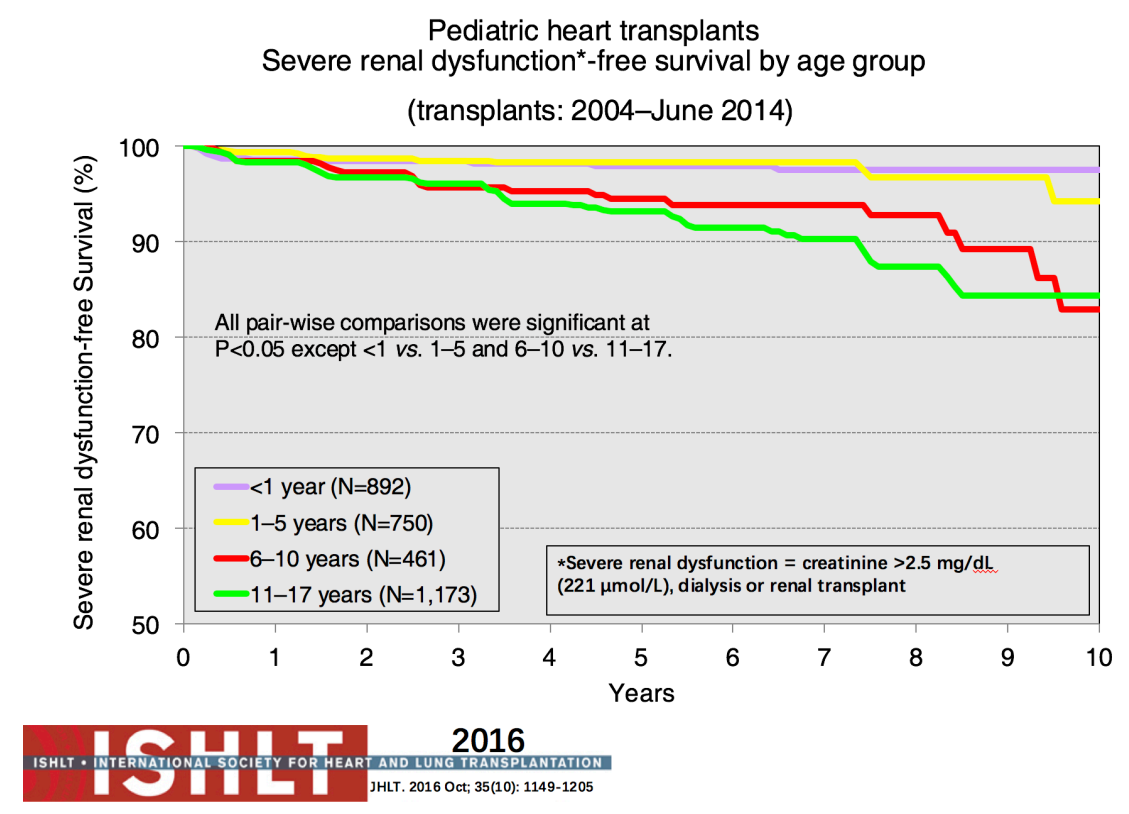

Figure 27 Freedom from severe renal dysfunction by age group in pediatric heart transplant recipients (from the registry of the International Society of Heart and Lung Transplantation. J Heart Lung Transplant 2016;35:1185-95).

including rejection and renal dysfunction (61).

\section{Other post-transplant complications}

Late renal dysfunction continues to be a potential risk after heart transplantation in children with some children progressing to dialysis and/or renal transplant (Figure 27). Risk factors from PHTS include black race, rejection with hemodynamic compromise, and decreased glomerular filtration rate at 1 -year post-transplant (62). Heart transplant programs should have protocols in place for proactive surveillance of renal function over time, practice renal-sparing strategies with all medication and therapy choices, encourage a minimum fluid intake daily, and have interventional strategies planned for mild, moderate and severely reduced renal function following heart transplantation.

Infections remain a challenge across the whole posttransplant period. Bacterial infections, especially in the first month post-transplant, were the most common cause of severe infections with an overall mortality of $34 \%$ (63). Cytomegalovirus has been purported to be possibly associated with acute rejection, CAV and graft loss, but this was not born out in the PHTS analysis should no demonstrable association with death or CAV (64). Regarding infections, the type, severity, risk factors, prophylaxis, management strategies, and impact on outcomes vary and are beyond the scope of this chapter though excellent summaries and guidelines exist in the literature (65).

\section{Psychosocial considerations}

\section{Functional status}

The most consistent data collection regarding functional status is within the US using the Lansky score and reported to the ISHLT registry (2). A recent expanded analysis showed that the vast majority of children who survive at least 1 year post-transplant have an excellent functional status with few limitations to daily life (66). Furthermore, children with intellectual disability are increasingly being considered for transplantation and an analysis from a similar dataset showed no difference in survival or acute rejection in addition to improvement in functional status following transplantation in 107 children with a diagnosis of intellectual disability, raising awareness of and supporting a shift in the historical practice of considering intellectual disability as a relative contraindication to transplantation (67).

\section{Non-adherence}

An awareness of and efforts to mitigate non-adherence 
are a significant part of the management of pediatric heart transplant recipients-especially during adolescence. The adolescent age range itself is independently associated with worse survival (1), with non-adherence being linked to late rejection and to high rates of death in adolescents (68). The ability and willingness to follow medical recommendations, biological factors, and the normal developmental challenges of adolescence that affect the ability to self-monitor and self-care all play a role in non-adherence (68-70). Identification and confirmation is challenging and can strain the relationships between the medical team, the adolescent and the family. A variability in trough levels of medications has been shown to be a marker for recurrent rejection and hospitalization after transplantation, and presumably nonadherence (71). Technologies aimed at targeting adolescents to help identify and manage non-adherence (including webbased and/or medical apps) continue to be developed (72). A coordinated approach between the transplant team, the adolescent, and the family must be in place, particularly when transitioning from pediatric to adult-based care.

\section{Transition}

Another area that has received tremendous attention in the recent past is the transition from pediatric-centered care to adult-centered care. Outcomes, especially rejection and graft loss, have been associated with a suboptimal transition process (73). It is clear now that transition needs to be a planned process that addresses the medical, psychological, and educational needs of adolescents and young adults with chronic physical and medical conditions. Also recognized more is the existence of a transition timeline; the process should be initiated with patients and families in their early teens and is an ongoing educational endeavor with a goal of providing uninterrupted health care. Focuses should include learning about their heart disease, medication doses and side effects, and signs and symptoms of infection and rejection. Skills to promote self-management are key and include learning to contact the pharmacy for medication refills, understanding when and how to contact the transplant team, practicing scheduling their own appointments and arranging transportation for clinic visits. Approaches to transition for continue to evolve, including the use of smart phones and other technologies (73).

\section{Limitations}

This keynote article was intended to provide a snapshot of the current state of pediatric heart transplantation. It summarizes key contemporary literature in the area. However, as is often the case both in transplantation and in pediatrics, the quality and strength of evidence is limited mostly to single center experiences and registry analyses with all of their inherent limitations. A handful of attempts at risk prediction models and/or decisionmaking algorithms are also included but are also based on retrospective registry-based analyses. Prospective, multicenter observational and/or interventional studies are sorely lacking in the field, though data from the cardiac consortium of the NIAID/NIH-sponsored CTOTC program (www.ctotc.org; U01AI077867) is forthcoming and will provide greater strength and level of evidence for many of the key challenges in the field.

\section{Conclusions}

Pediatric heart transplantation is standard of care for children with end-stage heart failure. The diverse age range, diagnoses, and practice variations continue to challenge the development of evidence-based practices and new technologies. Outcomes in the most recent era are excellent, with the biggest gap impacting both waitlist and overall survival being availability of mechanical support options for infants and patients with single ventricle physiology. Functional outcomes are excellent, but significant psychosocial challenges exist in relation to neurodevelopment, non-adherence, and transition from child-centered to adult-centered care. Pediatric heart transplantation continues to evolve in order to address the challenges of the diverse group of patients that reach endstage heart failure during childhood.

\section{Acknowledgements}

None.

\section{Footnote}

Conflicts of Interest: The author has no conflicts of interest to declare.

\section{References}

1. Rossano JW, Dipchand AI, Edwards LB, et al. The Registry of the International Society for Heart and Lung Transplantation: Nineteenth Pediatric Heart 
Transplantation Report-2016; Focus Theme: Primary Diagnostic Indications for Transplant. J Heart Lung Transplant 2016;35:1185-95.

2. Chambers DC, Yusen RD, Cherikh WS, et al. The Registry of the International Society for Heart and Lung Transplantation: Thirty-fourth Adult Lung And HeartLung Transplantation Report-2017; Focus Theme: Allograft ischemic time. J Heart Lung Transplant 2017;36:1047-59.

3. Dipchand AI, Kirk R, Mahle WT, et al. Ten years of pediatric heart transplantation: a report from the Pediatric Heart Transplant Study. Pediatric Transplantation 2013;17:99-111.

4. Almond CS, Thiagarajan RR, Piercey GE, et al. Waiting list mortality among children listed for heart transplantation in the united states. Circulation 2009;119:717-27.

5. Carlo WF, West SC, McCulloch M, et al. Impact of initial Norwood shunt type of young hypoplastic left heart syndrome patients listed for heart transplant: a multi-institutional study. J Heart Lung Transplant 2016;35:301-5.

6. Jeewa A, Manlhiot C, Kantor, PF, et al. Risk factors for mortality or delisting of patients from the pediatric heart transplant waiting list. J Thorac Cardiovasc Surg 2014;147:462-8.

7. Alsoufi B, Mahle WT, Manlhiot C, et al. Outcomes of heart transplantation in children with hypoplastic left heart syndrome previously palliated with the Norwood procedure. J Thorac Cardiovasc Surg 2016;151:167-74.

8. Almond CS, Hoen H, Rossano JW, et al. Development and validation of a major adverse transplant event (MATE) score to predict late graft loss in pediatric heart transplantation. J Heart Lung Transplant 2017. [Epub ahead of print].

9. Almond CS, Gauvreau K, Canter CE, et al. A riskprediction model for in-hospital mortality after heart transplantation in US children. Am J Transplant 2012;12:1240-8.

10. Feingold B, Webber SA, Bryce CL, et al. Comparison of listing strategies for allosensitized heart transplant candidates requiring transplant at high urgency: A decision model analysis. Am J Transplant 2015;15:427-35.

11. Schumacher KR, Almond C, Singh TP, et al. Predicting graft loss by 1 year in pediatric heart transplantation candidates. An analysis of the Pediatric Heart Transplant Study Database. Circulation 2015;131:890-8.

12. Zafar F, Castleberry C, Khan MS, et al. Pediatric heart transplant waiting list mortality in the era of ventricular assist devices. J Heart Lung Transplant 2015;34:82-8.

13. Jeewa A, Manlhiot C, McCrindle BW, et al. Outcomes with ventricular assist device versus extracorporeal membrane oxygenation as a bridge to pediatric heart transplantation. Artif Organs 2010;34:1087-91.

14. Blume ED, Rosenthal DN, Rossano JW, et al. Outcomes of children implanted with ventricular assist devices in the United States: First analysis of the Pediatric Interagency Registry for Mechanical Circulatory Support (PediMACS). J Heart Lung Transplant 2016;35:578-84.

15. Dipchand AI, Mahle WT, Tresler M, et al. Extracorporeal membrane oxygenation as a bridge to pediatric heart transplantation: Effect on post-listing and posttransplantation outcomes. Circ Heart Fail 2015;8:960-9.

16. Pietra BA, Kantor PF, Bartlett HL, et al. Early predictors of survival to and after heart transplantation in children with dilated cardiomyopathy. Circulation 2012;126:1079-86.

17. West LJ, Pollock-Barziv SM, Dipchand AI, et al: ABOincompatible heart transplantation in infants. $\mathrm{N}$ Engl J Med 2001;344:793-800.

18. West LJ, Karamlou T, Dipchand AI, et al. Impact on outcomes after listing and transplantation of a strategy to accept $\mathrm{ABO}$ blood group-incompatible donor hearts for neonates and infants. J Thorac Cardiovasc Surg 2006;131:455-61.

19. Dipchand AI, Pollock BarZiv SM, Manlhiot C, et al. Equivalent outcomes for pediatric heart transplantation recipients $\mathrm{ABO}$-blood group incompatible versus $\mathrm{ABO}$ compatible. Am J Transplant 2010;10:389-97.

20. Henderson HT, Canter CE, Mahle WT, et al. ABOincompatible heart transplantation: analysis of the Pediatric Heart Transplant Study (PHTS) database. J Heart Lung Transplant 2012;31:173-9.

21. Urschel S, Larsen IM, Kirk R, et al. ABO incompatible heart transplantation in early childhood: an international multicenter study of clinical experiences and limits. J Heart Lung Transplant 2013;32:285-92.

22. Conway J, Manlhiot C, Allain-Rooney T, et al. Development of donor-specific isohemagglutinins following pediatric ABO-incompatible heart transplantation. Am J Transplant 2012;12:888-95.

23. Irving CA, Gennery AR, Carter V, et al. ABOincompatible cardiac transplantation in pediatric patients with high isohemagglutinin titers. J Heart Lung Transplant 2015;34:1095-102.

24. Tait BD. Detection of HLA antibodies in organ transplant 
recipients - triumphs and challenges of the solid phase bead assay. Front Immunol 2016;7:570.

25. Mahle WT, Tresler MA, Edens RE, et al. Allosensitization and outcomes in pediatric heart transplantation. J Heart Lung Transplant 2011;30:1221-7.

26. Feingold B, Park SY, Comer DM, et al. Outcomes after listing with a requirement for a prospective crossmatch in pediatric heart transplantation. J Heart Lung Transplant 2013;32:56-62.

27. Feingold B, Webber SA, Bryce CL, et al. Comparison of listing strategies for allosensitized heart transplant candidates requiring transplant at high urgency: a decision model analysis. Am J Transplant 2015;15:427-35.

28. Webber S, Addonizio L, Blume E, et al. Pediatric Heart Transplantation Across a Positive Cross-Match Is Associated with High Rates of AMR but No Difference in Short Term Graft Loss, Dysfunction and Death (CTOTC-04 study). Am J Transplant 2016;16.

29. Zuckerman W, Feingold B, Zeevi A, et al. Solid Phase Assay Demonstrates High Rates of Allosensitization in Pediatric Heart Recipients. Am J Transplant 2016;16.

30. Castleberry C, Pruitt E, Ameduri R, et al. Risk stratification to determine the impact of induction therapy on survival, rejection and adverse events after pediatric heart transplant: a multi-institutional study. J Heart Lung Transplant 2017. [Epub ahead of print].

31. Rossano JW, Jeffries JL, Pahl E, et al. Use of sirolimus in pediatric heart transplant patients: a multi-institutional study from the Pediatric Heart Transplant Study Group. J Heart Lung Transplant 2017;36:427-33.

32. Auerbach SR, Kukreja M, Gilbert D, et al. Maintenance steroid use at 30 days post-transplant and outcomes of pediatric heart transplantation: a propensity matched analysis of the Pediatric Heart Transplant Study database. J Heart Lung Transplant 2015;34:1066-72.

33. Burckart GJ, Figg WD, Brooks MM, et al. Multiinstitutional study of outcomes after pediatric heart transplantation: candidate gene polymorphism analysis of ABCC2. J Pediatr Pharmacol Ther 2014;19:16-24.

34. Chen CK, Manlhiot C, Conway J, et al. Development and impact of de novo anti-HLA antibodies in pediatric heart transplant recipients. Am J Transplant 2015;15:2215-22.

35. Conway J, Dipchand AI. Challenges with sensitized recipients in pediatric heart transplantation. Clinics (Sao Paulo). 2014;69 Suppl 1:17-21.

36. Irving CA, Carter V, Gennery AR, et al. Effect of persistent versus transient donor-specific HLA antibodies on graft outcomes in pediatric cardiac transplantation. J
Heart Lung Transplant 2015;34:1310-7.

37. Dipchand AI, Webber S, Much K, et al. Impact of Newly Detected Donor Specific Anti-HLA Antibody in the First Year After Pediatric Heart Transplantation. J Heart Lung Transplant 2017;36:S78.

38. Gossett JG, Canter CE, Zheng J, et al. Decline in rejection in the first year after pediatric heart transplantation: a multi-institutional study. J Heart Lung Transplant 2010;29:625-32.

39. Everitt MD, Pahl E, Schechtman KB, et al. Rejection with hemodynamic compromise in the current era of pediatric heart transplant recipients: a multi-institutional study. J Heart Lung Transplant 2011;30:282-8.

40. Thrush PT, Pahl E, Naftel DC, et al. A multi-institutional evaluation of antibody-mediated rejection utilizing the Pediatric Heart Transplant Study database: incidence, therapies and outcomes. J Heart Lung Transplant 2016;35:1497-504.

41. Godown J, Harris MT, Burger J, et al. Variation in the use of surveillance endomyocardial biopsy among pediatric heart transplant centers over time. Pediatr Transplant 2015;19:612-7.

42. Stewart S, Winters GL, Fishbein MC, et al: Revision of the 1990 working formulation for the standardization of nomenclature in the diagnosis of heart rejection. J Heart Lung Transplant 2005;24:1710-20.

43. Berry GJ, Burke MM, Anderson C, et al. The 2013 International Society for Heart and Lung Transplantation Working Formulation for the standardization of nomenclature in the pathologic diagnosis of antibodymediated rejection in heart transplantation. J Heart Lung Transplant 2013;32:1147-62.

44. Miller CA, Fildes JE, Ray SG, et al. Non-invasive approaches for the diagnosis of acute cardiac allograft rejection. Heart 2013;99:445-53.

45. Tissot C, Pietra BA, Ivy DD, et al. Asymptomatic rejection diagnosed by surveillance endomyocardial biopsy: do we need to treat? J Heart Lung Transplant 2010;29:S171-2.

46. Everitt MD, Hammond ME, Snow GL, et al. Biopsydiagnosed antibody-mediated rejection based on the proposed International Society for Heart and Lung Transplantation working formulation is associated with adverse cardiovascular outcomes after pediatric heart transplant. J Heart Lung Transplant 2012;31:686-93.

47. Ameduri RK, Zheng J, Schechtman KB, et al. Has late rejection decreased in pediatric heart transplantation in the current era? A multi-institutional study. J Heart Lung Transplant 2012;31:980-6. 
48. Kobayashi D, Du W, L'ecuyer TJ. Predictors of cardiac allograft vasculopathy in pediatric heart transplant recipients. Pediatr Transplant 2013;17:436-40.

49. Mehra MR, Crespo-Leiro MG, Dipchand A, et al. International Society for Heart and Lung Transplantation working formulation of a standardized nomenclature for cardiac allograft vasculopathy-2010. J Heart Lung Transplant 2010;29:717-27.

50. Kindel SJ, Law YM, Chin C, et al. Improved detection of cardiac allograft vasculopathy: A multi-institutional analysis of functional parameters in pediatric heart transplant recipients. J Am Coll Cardiol 2015;66:547-57.

51. Potena L, Masetti M, Sabatino M, et al. Interplay of coronary angiography and intravascular ultrasound in predicting long-term outcomes after heart transplantation. J Heart Lung Transplant 2015;34:1146-53.

52. Mirelis JG, Garcia-Pavla P, Cavero MA, et al. Magnetic resonance for non-invasive detection of microcirculatory disease associated with allograft vasculopathy: intracoronary measurements validation. Rev Esp Cardiol (Engl Ed) 2015;68:571-8.

53. Tomai F, De Luca L, Petrolini A, et al. Optical coherence tomography for characterization of cardiac allograft vasculopathy in late survivors of pediatric heart transplantation. J Heart Lung Transplant 2016;35:74-9.

54. Mahle WT, Vincent RN, Berg AM, et al. Pravastatin therapy is associated with reduction in coronary allograft vasculopathy in pediatric heart transplantation. J Heart Lung Transplant 2005;24:63-6.

55. Chin C, Gamberg P, Miller J, et al. Efficacy and safety of atorvastatin after pediatric heart transplantation. J Heart Lung Transplant 2002;21:1213-7.

56. Greenway SC, Butts R, Naftel DC, et al. Statin therapy is not associated with improved outcomes after heart transplantation in children and adolescents. J Heart Lung Transplant 2016;35:457-65.

57. Jeewa A, Chin C, Pahl E, et al. Outcomes after percutaneous coronary artery revascularization procedures for cardiac allograft vasculopathy in pediatric heart transplant recipients: A multi-institutional study. J Heart Lung Transplant 2015;34:1163-8.

58. Shaddy RE, Revenaugh JA, Orsmond GS, et al. Coronary interventional procedures in pediatric heart transplant recipients with cardiac allograft vasculopathy. Am J Cardiol 2000;85:1370-2.

59. Tham EB, Yeung AC, Cheng CW, et al. Experience of percutaneous coronary intervention in the management of pediatric cardiac allograft vasculopathy. J Heart Lung
Transplant 2005;24:769-73.

60. Dipchand AI, Edwards LB, Kucheryavaya AY, et al. The registry of the International Society for Heart and Lung Transplantation: Seventeenth official pediatric heart transplantation report - 2014; focus theme: retransplantation. J Heart Lung Transplant 2014;33:985-95.

61. Conway J, Manlhiot C, Kirk R, et al. Mortality and morbidity after retransplantation after primary heart transplant in childhood: an analysis from the registry of the International Society for Heart and Lung Transplantation. J Heart Lung Transplant 2014;33:241-51.

62. Feingold B, Zheng J, Law YM, et al. Risk factors for late renal dysfunction after pediatric heart transplantation: a multi-institutional study. Pediatr Transplant 2011;15:699-705.

63. Rostad CA, Wehrheim K, Kirklin JK, et al. Bacterial infections after pediatric heart transplantation: epidemiology, risk factors and outcomes. J Heart Lung Transplant 2017;36:996-1003.

64. Mahle WT, Fourshee MT, Naftel DM, et al. Does cytomegalovirus serology impact outcome after pediatric heart transplantation? J Heart Lung Transplant 2009;28:1299-305.

65. Special Issue: The American Society of Transplantation Infectious Diseases Guidelines 3rd Edition. Am J Transplant 2013;13:1-371.

66. Peng DM, Zhang Y, Rosenthal DN, et al. Impact of heart transplantation on the functional status of US children with end-stage heart failure. Circulation 2017;135:939-50.

67. Wightman A, Bartlett HL, Zhao Q, et al. Prevalence and outcomes of heart transplantation in children with intellectual disability. Pediatr Transplant 2017;21:e12839.

68. Shellmer DA, Dabbs AD, Dew MA. Medical adherence in pediatric organ transplantation: what are the next steps? Curr Opin Organ Transplant 2011;16:509-14.

69. Oliva M, Singh TP, Gauvreau K, et al. Impact of medication non-adherence on survival after pediatric heart transplantation in the USA. J Heart Lung Transplant 2013;32:881-8.

70. Fine RN, Becker Y, De Geest S, et al. Nonadherence consensus conference summary report. Am J Transplant 2009;9:35-41.

71. Pollock-Barziv SM, Finkelstein Y, Manlhiot C, et al. Variability in tacrolimus blood levels increases the risk of late rejection and graft loss after solid organ transplantation in older children. Pediatr Transplant 2010;14:968-75. 
72. DeVito Dabbs A, Dew MA, Myers B, et al. Evaluation of a hand-held, computer-based intervention to promote early self-care behaviors after lung transplant. Clin Transplant 2009;23:537-45.

Cite this article as: Dipchand AI. Current state of pediatric cardiac transplantation. Ann Cardiothorac Surg 2018;7(1):3155. doi: 10.21037/acs.2018.01.07
73. Putschoegl A, Dipchand AI, Ross H, et al. Transitioning from pediatric to adult care after thoracic transplantation. J Heart Lung Transplant 2017;36:823-9. 